\title{
The OCO-2 oxygen A-band response to liquid marine cloud properties from CALIPSO and MODIS
}

Article

Published Version

Richardson, M., McDuffie, J., Stephens, G. L., Cronk, H. Q. and Taylor, T. E. (2017) The OCO-2 oxygen A-band response to liquid marine cloud properties from CALIPSO and MODIS. Journal of Geophysical Research: Atmospheres, 122 (15). pp. 8255-8275. ISSN 2169-8996 doi:

https://doi.org/10.1002/2017jd026561 Available at https://centaur.reading.ac.uk/72036/

It is advisable to refer to the publisher's version if you intend to cite from the work. See Guidance on citing.

To link to this article DOI: http://dx.doi.org/10.1002/2017jd026561

Publisher: American Geophysical Union

All outputs in CentAUR are protected by Intellectual Property Rights law, including copyright law. Copyright and IPR is retained by the creators or other copyright holders. Terms and conditions for use of this material are defined in the End User Agreement. 


\section{CentAUR}

Central Archive at the University of Reading

Reading's research outputs online 


\section{Journal of Geophysical Research: Atmospheres}

\author{
RESEARCH ARTICLE \\ 10.1002/2017JD026561 \\ Key Points: \\ - $\mathrm{O}_{2}$ A-band spectra inform about cloud \\ optical depth and photon path length \\ - We show that OCO-2 measurements \\ respond as expected to MODIS \\ optical depth and CALIPSO cloud top \\ pressure for liquid clouds \\ - A look-up table retrieval for optical \\ depth and cloud top pressure is \\ validated for OCO-2 radiances
}

Supporting Information:

- Supporting Information S1

- Data Set S1

Correspondence to:

M. Richardson,

markr@jpl.nasa.gov

\section{Citation:}

Richardson, M., J. McDuffie,

G. L. Stephens, H. Q. Cronk, and

T. E. Taylor (2017), The OCO-2 oxygen

A-band response to liquid marine cloud properties from CALIPSO and MODIS,

J. Geophys. Res. Atmos., 122, 8255-8275, doi:10.1002/2017JD026561.

Received 25 JAN 2017

Accepted 19 JUL 2017

Accepted article online 25 JUL 2017

Published online 10 AUG 2017

(2)17. American Geophysical Union. All Rights Reserved.

\section{The OCO-2 oxygen A-band response to liquid marine cloud properties from CALIPSO and MODIS}

\author{
Mark Richardson $^{1}$ iD, James McDuffie ${ }^{1}$ iD, Graeme L. Stephens ${ }^{1,2}$ iD, Heather Q. Cronk ${ }^{3}$ iD, \\ and Tommy E. Taylor ${ }^{3}$ \\ ${ }^{1}$ Jet Propulsion Laboratory, California Institute of Technology, Pasadena, California, USA, ${ }^{2}$ Department of Meteorology, \\ University of Reading, Reading, UK, ${ }^{3}$ Cooperative Institute for Research in the Atmosphere, Colorado State University, Fort \\ Collins, Colorado, USA
}

Abstract Spectra of reflected sunlight in the oxygen A-band contain information about cloud properties such as cloud top pressure, optical depth, and pressure thickness. Here we show, for the first time, that high-spectral-resolution A-band Orbiting Carbon Observatory-2 (OCO-2) spectra respond largely as simulated to the optical properties of water clouds over ocean during November $2015(N=184,318)$ using input cloud properties from the Moderate Resolution Imaging Spectroradiometer (MODIS) on Aqua and the Cloud-Aerosol Lidar with Orthogonal Polarization on Cloud-Aerosol Lidar and Infrared Pathfinder Satellite Observation (CALIPSO). In A-band continuum channels the standard deviation of simulated minus observed radiance is $\pm 37 \%$. Selecting horizontally homogeneous clouds to mitigate three-dimensional cloud effects and collocation error with the other satellites, the standard deviation of the residuals is reduced to $\pm 18 \%$. Using a look-up table developed from simulations, OCO-2's estimated cloud top pressure for low clouds $\left(P_{\text {top }}>680 \mathrm{hPa}\right)$ has a standard deviation of $\pm 61 \mathrm{hPa}$ relative to CALIPSO retrievals, and bias is dependent on assumed cloud pressure thickness, with our smallest value being $-5 \mathrm{hPa}$. Versus MODIS optical depth, the standard deviation is \pm 9.0 and the bias is -2.0 , although these shrink for clouds of $\tau<30$. These values include collocation error between the different satellites, meaning that they place an upper bound on the OCO-2 retrieval uncertainty. The theoretical precision limit from OCO-2's instrumental uncertainty is shown to be $\pm 2.4 \mathrm{hPa}$ in above-cloud path and $\pm 0.2 \%$ in optical depth for a two-channel retrieval. Options for retrieving cloud optical depth, cloud top pressure, and pressure thickness are discussed in the context of a formal OCO-2 cloud property retrieval.

\section{Introduction}

Cloud albedo is largely determined by optical depth, and upward longwave emission by the temperature at cloud top pressure, so these properties are important for determining the radiative impacts of clouds. Additionally, knowledge about cloud pressure thickness provides constraints for physical processes related to cloud formation, which are of interest because uncertainty in low-cloud shortwave feedback explains much of the spread in modeled climate sensitivity, or global warming in response to a given heating [Bony and Dufresne, 2005; Sherwood et al., 2014]. Constraints on low-cloud properties inform the development of model parameterizations of these clouds and therefore improve climate projections.

High spectral resolution measurements of reflected sunlight in the oxygen A-band contain information about cloud optical depth, cloud top pressure, and cloud pressure thickness [Fischer and Grassl, 1991; Koelemeijer et al., 2001; Rozanov, 2004; Stephens and Heidinger, 2000]. Clouds reflect sunlight in the oxygen A-band, and the observed radiances are related to cloud properties in two main ways: first, in window channels where there is very weak absorption, radiance is controlled by solar zenith angle, surface albedo, and cloud optical depth. Second, the ratio of observed radiance within an absorption band to that observed outside the absorption band is related to the photon path length and is therefore a measure of both cloud top pressure and cloud pressure thickness. This is because photons reflected from the cloud must travel to the cloud top and back again, such that a higher cloud top pressure (lower altitude) results in greater photon path length and absorption. Regarding geometric thickness, photons arriving at cloud top will experience some path through the cloud and in an optically thick cloud will tend to be multiply scattered until they escape. The total photon path is magnified when the droplets are further apart, which for a cloud with a given optical depth and droplet distribution may be understood as an increase in the geometric thickness. Increases in geometric thickness therefore relate to increased photon path lengths and increased absorption, which has been 
exploited in various proposals for retrieving geometric thickness using the A band [O'Brien and Mitchell, 1992; Stephens and Heidinger, 2000; Ferlay et al., 2010].

The spectral responses to cloud top pressure and cloud pressure thickness are strongly correlated, and so disaggregating them requires tight constraints on one or very high spectral resolution that allows more precise determination of photon-path length changes due to multiple scattering within the cloud and the differential broadening of absorption bands through the atmosphere.

A-band cloud top pressure retrievals rely on reflected sunlight and so provide information that is independent of active lidar (e.g., Cloud-Aerosol Lidar and Infrared Pathfinder Satellite Observation (CALIPSO)), radar (e.g., CloudSat), or infrared emission (e.g., MODIS). The A band offers benefits in particular for optically thick low clouds, where lidar cannot retrieve optical depth due to signal attenuation and infrared cloud top pressure retrievals have large uncertainty due to the diffuse cloud tops and complex temperature profiles which commonly feature inversions [Holz et al., 2008; Baum et al., 2012]. Furthermore, CloudSat radar retrievals suffer from surface clutter in low marine clouds [Huang et al., 2012]. The pressure thickness of low clouds is not reliably retrieved by any current satellite product, active or passive.

Among A-band sensors OCO-2 has substantial advantages. It has a much higher spectral resolution than almost all previous A-band instruments with 1016 channels from 758 to $773 \mathrm{~nm}$ and an average channel full width at half maximum (FWHM) of $0.04 \mathrm{~nm}$. Higher spectral resolution leads to improved sampling of the range of oxygen absorption. This spectral sampling is far higher than that used in or suggested for use in cloud-property retrievals such as those from ADEOS-POLDER [Vanbauce et al., 1998], PARASOLPOLDER [Sneep et al., 2008], IRS3-MOS [Preusker et al., 2007], ENVISAT-Medium-Resolution Imaging Spectrometer (MERIS) [Preusker and Lindstrot, 2009], and ERS-2-GOME [Koelemeijer et al., 2001]. These retrievals typically use 2-4 channels with instrumental FWHM of 0.4-0.7 nm, resulting in cloud top pressures within $\pm 30-100 \mathrm{hPa}$ of those reported by other methods such as radiosondes and passive infrared retrievals. In addition, there are substantial biases as these methods typically retrieve a single cloud pressure corresponding to a location within the cloud, rather than attempting to disaggregate cloud top pressure and cloud pressure thickness.

One other satellite carries an instrument whose A-band spectral resolution is similar to that of OCO-2: the Japanese Greenhouse Gases Observing Satellite (GOSAT) [Kuze et al., 2009; Yoshida et al., 2011] However, OCO-2 samples at a higher spatial resolution ( $<2.25 \mathrm{~km}$ per sounding versus $10.5 \mathrm{~km}$ for GOSAT), enabling finer-scale analysis. OCO-2 is also in the Afternoon-Train (A-Train), a constellation of Earth observation satellites in Sun-synchronous orbit, which allows data from multiple sensor types to be combined to allow a fuller assessment of the response of the A-band instrument to clouds.

Example high-resolution OCO-2 spectra are shown in Figure 1a, as simulated for a low and optically thin cloud (blue), low-thick cloud (green), and high-thick cloud (red) over ocean. In the A-band, ocean albedo is low meaning that the signal in this figure is dominated by cloud reflection, but optically thin clouds over brighter surfaces would show differences due to surface reflection. Interpreting the spectra is more intuitive when they are reorganized according to the mean oxygen absorption coefficient of the channel as in Figure $1 \mathrm{~b}$ [Stephens and Heidinger, 2000]. In this paper we sort spectra by considering the mean cloudy scene radiance in November 2015 and then ranking the channels from brightest to least bright separately for each OCO-2 across-track sounding position. This approximates the ranking by $\mathrm{O}_{2}$ absorption, and these rankings are then used to sort each spectrum. Higher optical depth results in a brighter spectrum with the largest absolute increase in the continuum where $\mathrm{O}_{2}$ absorption is weak. The response to cloud top pressure change is visible within the absorption bands in Figure $1 \mathrm{~b}$. The higher cloud (lower $P_{\text {top, }}$ red line) becomes brighter in these bands due to less above-cloud absorption. By combining continuum radiances for optical depth and absorption band radiances for photon path length a joint $\tau-P_{\text {top }}$ retrieval is possible. Sanghavi et al. [2015] assess the effect of cloud properties on A-band radiances by studying radiative transfer model output for theoretical cloud cases and their paper provides detailed theoretical explanations for the radiance responses expected and observed here.

Here we examine the response of OCO-2 A-band radiances to cloud properties measured by other A-Train sensors. Optical depth and droplet effective radius are provided by the Moderate Resolution Imaging Spectroradiometer (MODIS) instrument on Aqua and cloud top pressure from the Cloud-Aerosol Lidar with Orthogonal Polarization (CALIOP) instrument on CALIPSO. 

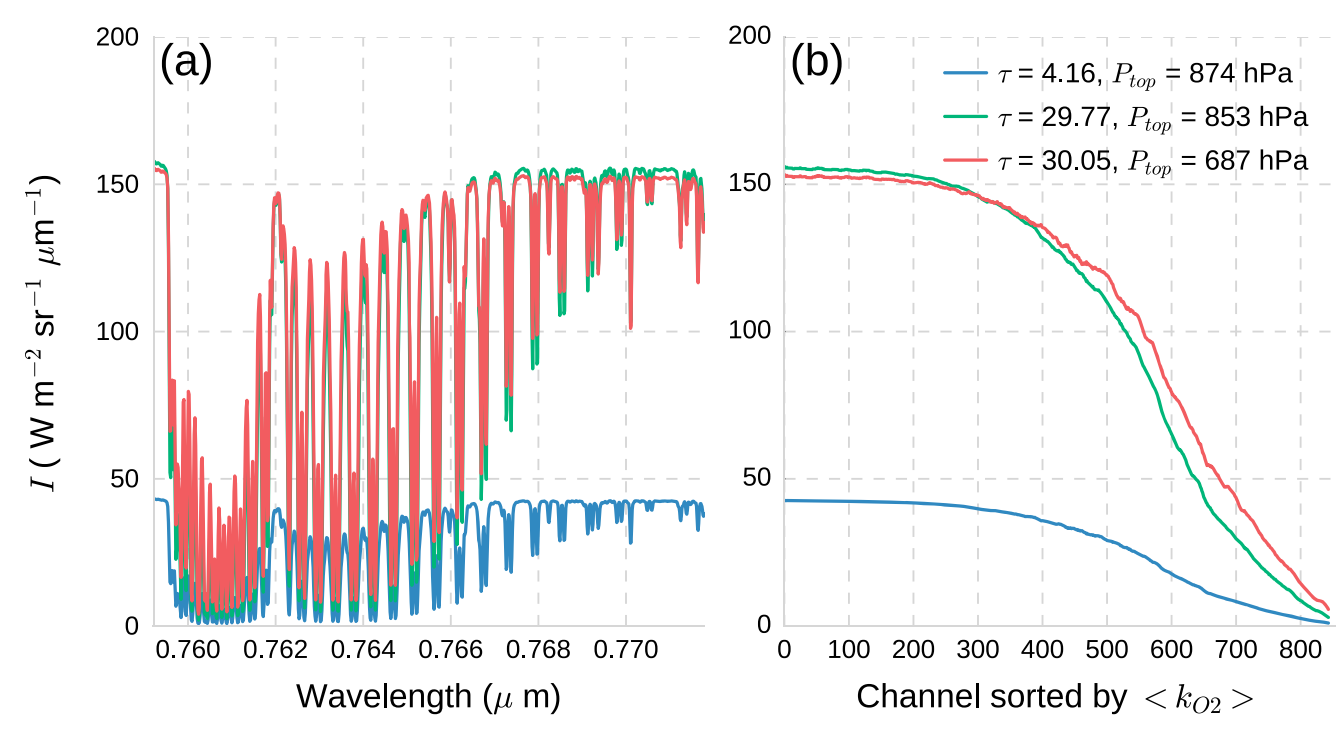

Figure 1. Example of simulated OCO-2 spectra for a low, optically thin cloud (blue), low optically thick cloud (green), and a high optically thick cloud (red) over a water surface with albedo $=0.1$. Input geometric thickness is calculated from equation (3) assuming effective droplet radius of $12 \mu \mathrm{m}$ (a) Spectra as a function of wavelength; (b) OCO-2 channels sorted by the mean channel brightness during November 2015. This is an approximation of ranking by $\mathrm{O}_{2}$ absorption coefficient. The exact ranking depends on precise atmospheric conditions and cloud height and so changes for each sounding.

We simulate OCO-2 radiances using the radiative transfer model used in the OCO-2 Level 2 Full Physics algorithm with cloud properties taken from collocated MODIS-CALIOP scenes from November 2015 [Taylor et al., 2016] and validate them using OCO-2 measurements. We then use the simulated relationships between cloud properties and radiances to develop look-up tables for $P_{\text {top }}$ and $\tau$ and validate them by comparing their predictions with collocated MODIS and CALIPSO data. These look-up tables will be used in future work to provide the first-guess properties necessary in a formal Bayesian retrieval that is under development. In this case we focus on retrievals of single-layer liquid clouds over ocean. This is motivated by how uncertainties in oceanic low-cloud reflection explain much of the spread in modeled global warming [Slingo, 1990; Webb et al., 2006; Brient et al., 2016]. This choice also avoids reflective land surfaces and complex ice-particle phase functions.

This paper is structured as follows: Section 2 describes the observational data, radiative transfer model, and analysis procedures; section 3 presents a direct comparison of simulated and observed spectra along with the determined sensitivity of OCO-2 radiances to cloud properties. Section 4 discusses the results in the context of a formal error analysis, and section 5 concludes.

\section{Methods and Data}

Section 2.1 identifies the primary OCO-2, MODIS, CALIPSO, and European Centre for Medium-Range Weather Forecasts (ECMWF) data sets used in this analysis and overviews relevant details of the OCO-2 mission. Section 2.2 introduces the collocated MODIS-CALIPSO-OCO-2 data sets, highlights potential uncertainties, and lists the sample selection criteria. Section 2.3 briefly describes the radiative transfer model and how the atmospheric profiles were constructed for the simulations. Section 2.4 describes the primary analysis techniques for radiance response to cloud properties, and section 2.5 describes the way in which the lookup tables for first-guess cloud-property retrievals were developed and validated.

\subsection{Observational Data}

OCO-2 leads the A-Train constellation, whose satellites follow a Sun-synchronous orbit with a 16 day repeat cycle at the equator where crossing time is near 13:30 in the ascending node at an altitude of $705 \mathrm{~km}$. The OCO-2 reference ground track (RGT) is identical to that of CALIPSO and CloudSat, displaced $217.3 \mathrm{~km}$ to the east of the World Reference System (WRS)-2 RGT [Taylor et al., 2016]. Aqua follows the WRS-2 RGT and 
carries the MODIS instrument whose wide field of view includes the OCO-2 track, while the CALIPSO satellite carries the nadir-view CALIOP lidar and follows the same RGT as OCO-2.

OCO-2's primary mission is to determine atmospheric column abundance of $\mathrm{CO}_{2}$ in parts per million (XCO2 [Crisp, 2008; Crisp et al., 2016; Eldering et al., 2016; Osterman et al., 2016]), although it also samples wavelengths that allow determination of solar-induced fluorescence (SIF) resulting from photosynthesis, and so reports both XCO2 and SIF products [Frankenberg et al., 2014]. It carries three co-bore-sighted, imaging, grating spectrometers collecting high-resolution spectra reported at 1016 channels in each of the $\mathrm{O}_{2} \mathrm{~A}$-band (near $765 \mathrm{~nm}$ ), weak $\mathrm{CO}_{2}$ band $(1610 \mathrm{~nm})$, and strong $\mathrm{CO}_{2}$ band $(2060 \mathrm{~nm})$. The $\mathrm{O}_{2} \mathrm{~A}$-band is primarily used for cloud filtering and the determination of surface pressure so as to convert measured $\mathrm{CO}_{2}$ amount to XCO2. Scenes that are even modestly contaminated by clouds or aerosols are not processed by the L2 algorithm because the retrieval of XCO2 is highly sensitive to scattering.

OCO-2 is flying flight-spare focal plane array (FPA) detectors from the original OCO mission, which failed at launch. The FPA was delivered in 2006, and prior to orbit a number of the pixels failed or respond anomalously to light or thermal changes [Osterman et al., 2016]. In this analysis we only use data from pixels that are undamaged for all measurements, reducing the number of channels from 1016 to 853 . Defining continuum channels as those where there is $\geq 99 \%$ of the mean radiance of the single brightest channel, our results are not strongly affected by pixel damage as we have 156 remaining continuum channels and only lose nine absorption-band channels.

OCO-2 operates in a pushbroom fashion with a single frame consisting of eight soundings, which are lined up side-by-side with respect to the orientation of the spacecraft and in nadir view have a ground footprint of approximately $1.3 \mathrm{~km}$, meaning the full swath width is approximately $10 \mathrm{~km}$ [Crisp et al., 2016]. In the original design the spacecraft was oriented with its photovoltaics orthogonal to the incoming sunlight in order to maximize power generation. A later engineering issue related to the orientation of spectrometer gratings required an in-flight reorientation to approximately $30^{\circ}$ from the original flight plan [Crisp et al., 2016].

OCO-2 has three main measurement modes: target (TG), glint (GL), and nadir (ND). Target viewing fixes the instrument on a desired ground point as the satellite passes overhead, such as a station in the Total Carbon Column Observing Network in order to perform calibration or validation. Glint mode points the instrument toward the glint spot in order to maximize surface reflection and increase signal for the XCO2 retrieval over oceans. Nadir viewing measures straight down, which is preferred for XCO2 retrievals over brighter land surfaces, and also increases the likelihood of viewing between broken cloud fields by minimizing the field of view. Aside from occasional orbits in TG mode, the current flight mode procedure alternates GL and ND orbits, with some orbits that pass mostly over ocean always operating in GL viewing [Crisp et al., 2016].

We select nadir-view orbits in order to match up with the nadir-view CALIPSO lidar, using L1bSc Version 7 data for November 2015 (OCO-2 orbits 7111-7522). The primary observable is the reported A-band radiance (variable SoundingMeasurements/radiance_o2 in the L1bSc product nomenclature), although a number of ancillary data were also used.

The radiative transfer simulations also require meteorological inputs such as vertical profiles of temperature and specific humidity. All necessary meteorological data were taken from the OCO-2 auxiliary ECMWF files, which contain the short-term ECMWF forecast data interpolated in time and space to each measurement point. We use the MODIS collocation files based on Aqua product MYD06_L2 [Platnick et al., 2015] which provides input cloud properties at $1 \mathrm{~km}$ spatial resolution, henceforth referred to as MYD061KM. We use the cloud top effective radius, cloud optical thickness, and cloud phase determined from optical properties. From CALIPSO we use the $01 \mathrm{kmCLay}$ product for the cloud top pressure [Vaughan et al., 2009].

\subsection{Collocated Data and Sample Selection Criteria}

The orbital geometry of the A-Train satellites prevents exact collocation: OCO-2 leads the A-Train and is followed within approximately $7.5 \mathrm{~min}$ by both Aqua and CALIPSO, and data collocation matches the nearest neighbor pixel based on minimizing the distance between each instrument's footprint, using a previously described A-Train algorithm [Savtchenko et al., 2008] that has been modified for the OCO-2 ground track [Taylor et al., 2016]. The $2330 \mathrm{~km}$ MODIS cross-track swath ensures that all nadir-view OCO-2 soundings fall 
within the MODIS field of view. Since the OCO-2 RGT is approximately $200 \mathrm{~km}$ to the east of the MODIS instrument, there is a consistent difference in viewing angle which, in some cases, may lead to collocation error. For example, parallax may shift apparent cloud location and any viewing-angle-dependent biases in MODIS cloud properties will be present in this matchup data. Here "collocation error" is taken to mean any difference in the field of view observed by OCO-2, MODIS, and CALIPSO due to viewing geometry, viewing time, or location error.

The matchup files report the matchup distance between each retrieval and the OCO-2 sounding, and we select only those OCO-2 soundings for which the CALIPSO matchup distance is under $1 \mathrm{~km}$. This reduces errors introduced in our simulations due to cloud variability on 1-10 km horizontal scales, but we are not able to account for the order of magnitude difference between the OCO-2 and CALIOP field of view, where CALIOP native measurement resolution is approximately $70 \mathrm{~m}$, with one measurement each $333 \mathrm{~m}$ along track [Vaughan et al., 2009; Winker et al., 2010].

The relative positions of the OCO-2 and CALIPSO satellites vary substantially within their control boxes. Particular effort was made to keep the satellites well aligned during the nominal mission lifetime, but for our requirements matchup quality varies with time. The number of soundings with matchup distance under $1 \mathrm{~km}$ varies with month, and the location of CALIPSO measurements within the OCO-2 swath also varies. The ideal case is where matched up CALIPSO measurements are spread across each of the OCO-2 soundings, but in some months there is a predominance of measurements at the edge of the OCO-2 swath where OCO-2 sensor calibration is most difficult. Our choice of November 2015 was based on maximizing valid retrievals across multiple soundings and accounting for potential instrumentation issues (Crisp, personal communication). The specific criteria applied to the data sets used are listed in Table S1 in the supporting information.

Some combinations of properties caused computational failures in the radiative transfer model: $N=184,318$ soundings matched our criteria and were successfully simulated. It is worth noting that not only are nadir soundings over ocean rarely used for the OCO-2 XCO2 retrieval due to insufficient signal-to-noise ratio, but that the XCO2 retrieval is only performed in cases where the algorithm is confident of clear-sky conditions. This paper therefore exploits a data set that was previously untapped.

\subsection{Simulated Radiances}

Radiances are simulated using the OCO-2's Level 2 Full Physics retrieval's radiative transfer model (henceforth L2RTM [Boesch et al., 2015]). Fundamentally, this model follows the eigenvector method of solving the radiative transfer equation [Flatau and Stephens, 1988] and is based on a version of the LIDORT linearized discreteordinate radiative transfer model [Spurr et al., 2001; Spurr, 2002] modified with a second order of scattering technique called 2OS [Natraj and Spurr, 2007]. This modification reduces the computational burden of determining multiple scattering effects on the polarized elements of the Stokes vector by 2 orders of magnitude versus fully vectorized multiple-scattering computations. The results for OCO-2 viewing geometries have been tested against such calculations from the vectorized VLIDORT [Spurr, 2006].

The model is semispherical, accounting for changes in atmospheric path length of the initial beam due to Earth's curvature, but is otherwise horizontally homogeneous. The angular integrations required for scattering are approximated via quadrature with high-accuracy calculations using 16 quadrature angles (i.e., streams) for 5-20 of the wavelengths in each band. Radiative transfer in the remaining wavelengths is calculated using simplified two-stream scalar radiative transfer with a polarized single-scattering code. From the combination of these results, high-accuracy results at all wavelengths can be reconstructed [O'Dell, 2010].

We use the OCO-2 instrument line shapes (ILS) for November 2015, which are defined for each of the eight sounding positions within a frame. Atmospheric molecular absorption is determined using the same absorption coefficient (ABSCO) tables as for the v7 OCO-2 operational product except for $\mathrm{O}_{2}$ which has a new version OCO-2 ABSCO v5 [Drouin et al., 2017]; this is the selected ABSCO for the v8 OCO-2 operational products. The atmospheric profiles for the radiative transfer calculations are input on 20 pressure levels, linearly interpolated from $1 \mathrm{hPa}$ to the surface. Meteorological information is taken from the standard OCO-2 matched ECMWF files and interpolated from the native 137 levels.

The cloud is assumed to consist of a single homogeneous layer of liquid water droplets. Cloud-top pressure is taken from CALIOP, optical depth and effective droplet radius from MODIS. Consistently reliable cloud 
pressure thickness is not available in any collocated product, however, so we use approximations developed for marine stratocumulus clouds based on assumed adiabatic profiles.

We begin with a relationship between cloud liquid water path (LWP) and geometrical thickness $H$ [Brenguier et al., 2000]:

$$
\operatorname{LWP}=\frac{1}{2} C_{w} H^{2}
$$

where $C_{w}$ is the moist adiabatic condensate coefficient and for marine stratocumulus was reported as lying in the range 1-2.5 $\times 10^{-3} \mathrm{~g} \mathrm{~m}^{-4}$ [Brenguier, 1991]. Next, we use the relationship between liquid water path, optical depth $\tau$, and effective droplet radius $r_{\text {eff }}$ from [Szczodrak et al., 2001]:

$$
\mathrm{LWP}=\tau r_{\mathrm{eff}} \frac{10 \rho_{w}}{9 Q_{\mathrm{ext}}}
$$

which differs by a factor of 6/5 from the classical relationship of Stephens [1978] because it uses an adiabatic cloud model instead of a vertically homogeneous model, which better represents the marine stratiform cloud case [Wood and Hartmann, 2006; Borg and Bennartz, 2007]. By combining equations (1) and (2) we obtain an equation for the geometrical thickness of

$$
H=\sqrt{\frac{5 \rho_{w} \tau r_{\mathrm{eff}}}{9 Q_{\mathrm{ext}} C_{w}}}
$$

We then assume a pressure scale height of $8 \mathrm{~km}$ to relate $H$ to cloud pressure thickness $\Delta P$, and for our base case we use $Q_{\text {ext }}=2$ and $\rho_{w}=1000 \mathrm{~kg} \mathrm{~m}^{-3}$, with a value of $C_{w}=1.9 \times 10^{-3} \mathrm{~g} \mathrm{~m}^{-4}$ based on previous work [Brenguier et al., 2000]. There are uncertainties in $\tau$ and $r_{\text {eff, }}$ and $C_{w}$ depends on temperature, with a range of $1.0-2.5 \times 10^{-3} \mathrm{~g} \mathrm{~m}^{-4}$ for temperatures $0-40^{\circ} \mathrm{C}$ [Brenguier, 1991]. In addition, this relationship is based on assumptions appropriate for marine stratocumulus clouds and is unlikely to be representative of many of the clouds in our sample. However, such an assumption is necessary given the lack of available global cloud thickness information. Differences between our assumed cloud thickness and the real cloud thicknesses should be expressed as differences in the modeled and simulated photon path lengths and so cause differences in simulated radiances in the absorption band. We address this uncertainty by performing three sets of radiative transfer simulations in which the above-derived cloud pressure thickness is scaled by $[0.5,1.0,4.0]$. In general, we report the default results with scale factor 1.0 in the manuscript and present results with different scale factors in the supporting information, unless specified. This allows us to estimate the robustness of our results to the cloud thickness assumption. Within the cloud, droplets are represented by a gamma distribution with the MODIS effective radius derived from optical properties, and single scattering properties are determined from Mie theory.

Cloud layers in this implementation of the radiative transfer are determined by interpolating the extinction provided at one level to the neighboring levels, and then assuming that the layers between these three levels are filled with a homogeneous cloud. We therefore assign three levels, equidistant in terms of pressure, to each cloud. The top level pressure is that given as cloud top pressure from CALIOP, the full pressure difference between the top and bottom levels is that determined from our scaled equation (3), and the extinction coefficient is assigned to the central level such that the calculated optical depth matches that derived from MODIS. If the derived cloud bottom is below the ECMWF surface pressure, then it is lifted to $20 \mathrm{hPa}$ above the surface without moving the cloud top, while ensuring that the determined optical depth still matches the MODIS value.

Pressure levels were first determined by linearly interpolating from $1 \mathrm{hPa}$ to the ECMWF surface pressure, then assigning the cloud to the nearest pressure levels subject to the constraint that at least 2 levels must lie above or below the cloud. Once the cloud levels are assigned, the levels above and below the clouds are recalculated by interpolating linearly from $1 \mathrm{hPa}$ to cloud top pressure, and from the surface pressure to cloud-bottom pressure. For a set of sample cases, the above-cloud oxygen optical depth calculated using this pressure structure was found to be within $1 \%$ of that calculated using the original 137-level ECMWF input profiles for all channels. 


\subsection{Comparison Methodology}

We begin by simulating OCO-2 A-band spectra for each set of MODIS-CALIPSO cloud properties as described in section 2.3 and then reorganize each spectrum according to the mean simulated radiance of that channel, from brightest to least bright. As $\mathrm{O}_{2}$ absorption dominates at A-band wavelengths, this approach is similar to ranking by the path-weighted $\mathrm{O}_{2}$ absorption coefficient. We do not formally rank by $\mathrm{O}_{2}$ absorption coefficient because this varies throughout the atmosphere and each individual sounding experiences a different set of path-weighted channel oxygen absorption coefficients.

All spectra are grouped by their across-track position, and with eight soundings per frame, resulting in eight data sets. This is done to separately account for each sounding position's different instrument line shapes (ILS). We then average all simulated spectra to produce a single mean spectrum for each sounding position. From these spectra, we obtain eight sets of rankings required to organize them from most to least bright, and then each individual observed or simulated spectrum is sorted using the rankings appropriate for its acrosstrack position.

In order to mitigate intersounding differences in path length and absorption coefficient, we take 10-channel averages and refer to these as superpixels, resulting in 85 superpixels of 10 channels and 1 of 3 channels. By sorting channels prior to smoothing, the advantage of OCO-2's spectral resolution is not notably degraded, although there are other minor issues to consider such as instrument dispersion, albedo changes across the band and solar lines (C.W. O'Dell, personal communication).

In addition to absolute radiance, we are interested in the response of observable properties (e.g., radiance) to cloud properties (e.g., optical depth). Standard retrieval methods such as optimal estimation traditionally use numerical partial derivatives, but these cannot be directly observed. Here we determine sensitivities by regressing observed or simulated OCO-2 radiances for each superpixel against the MODIS and CALIPSO cloud properties. The gradient of the regression line is taken to be the relevant sensitivity, and we then estimate the measurement precision of a state vector element $\sigma_{x, j}$ from a superpixel cloudproperty retrieval as

$$
\sigma_{x, j}=\left(\frac{\partial y_{i}}{\partial x_{j}}\right)^{-1} \sigma_{y, i}
$$

where $\frac{\partial y_{i}}{\partial x_{j}}$ is our sensitivity, which we estimate from the gradient of our regression fit and $\sigma_{y, i}$ is the OCO-2 $\mathrm{L} 1 \mathrm{bSc}$ measurement uncertainty. This $\sigma_{y, i}$ is measurement precision only, and error introduced by other factors is much larger. To place an upper bound on the total error in retrievals of cloud optical depth and cloud top pressure, we therefore apply a simple look-up table-based retrieval developed using the simulated radiance responses and validate it using the collocated MODIS-CALIPSO-OCO-2 observations.

\subsection{Theoretical Foundation of Cloud Property Retrieval}

We develop two independent look-up tables, one for cloud $\tau$ and another for cloud top pressure. The $\tau$ lookup table relies on the radiance from a single superpixel in the continuum, and the $P_{\text {top }}$ retrieval uses the ratio of the radiance of one superpixel to that of a continuum superpixel. We calculate look-up tables using all of the superpixels and show their relative performance, but our final look-up-based retrieval uses two superpixels that are selected to minimize error statistics against collocated MODIS $\tau$ and CALIPSO $P_{\text {top. }}$

Optical depth is the dominant factor in determining measured reflectance, and to account for illumination angle, we approximate the cloud as a Lambertian reflector and divide measured radiance by the cosine of the solar zenith angle, $\cos \left(\theta_{\mathrm{SZA}}\right)=\mu$. For each superpixel we then bin the simulated $I / \mu$ by cloud optical depth and take the bin-median $I / \mu$ and $\tau$ as our look-up table entry.

For our $P_{\text {top }}$ look-up table we use the ratio $I / I_{c}$ where $I_{c}$ is the continuum radiance, in this case the radiance in the first ranked superpixel. If the absorption in this superpixel is dominated by $\mathrm{O}_{2}$, then from the BeerLambert law, for radiance in a channel $i$ :

$$
\frac{l_{i}}{I_{c}}=\exp \left(-\tau_{i, \mathrm{O} 2}\right)
$$




\section{November 2015}
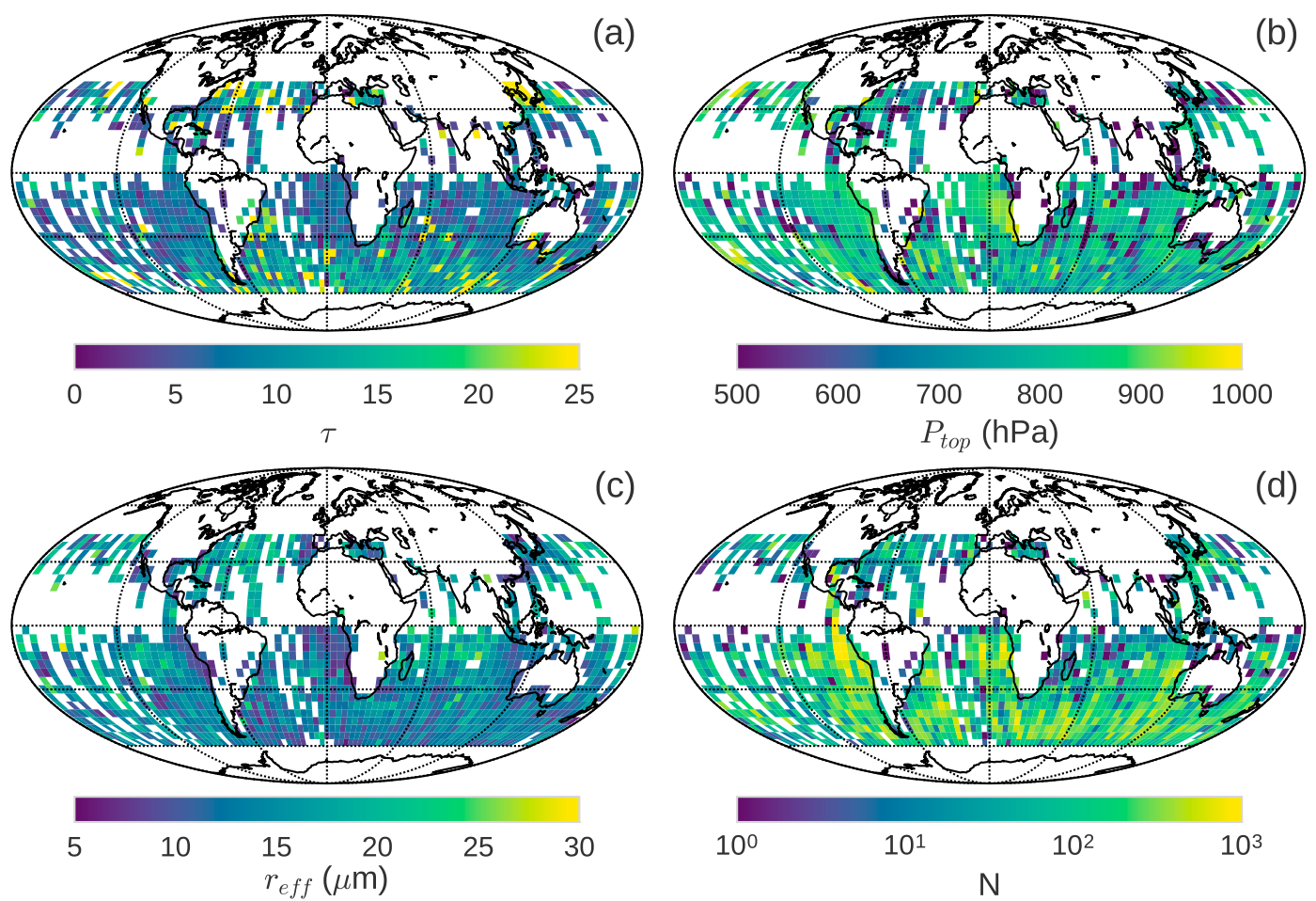

Figure 2. Maps of cloud sample properties in $4 \times 4^{\circ}$ latitude-longitude grid cells from OCO- 2 nadir-view measurements in November 2015, for cloudy scenes where CALIPSO retrieves a cloud top pressure and MODIS retrieves a liquid cloud. (a) Mean MODIS optical depth, (b) mean CALIOP cloud top pressure, (c) mean MODIS droplet effective radius, and (d) number of valid retrievals per grid cell (log scale).

where $\tau_{i, 02}$ is the oxygen optical depth in channel $i$, and it is assumed that continuum absorption is negligible, i.e., $\tau_{c, \mathrm{O} 2} \cong 0$. Given the small wavelength range, we assume that scattering and absorption by clouds are similar between the two superpixels. We can split the optical depth into above-cloud $\left(\tau_{\mathrm{O} 2}\right)$ and within-cloud $\left(\tau_{\mathrm{O} 2, c}\right)$ components and take the natural logarithm:

$$
-\ln \left(\frac{I_{i}}{I_{c}}\right)=\tau_{02, c}+\tau_{02}
$$

We then express the optical depths in terms of a profile of oxygen absorption coefficient $k$ integrated along some path from 0 to $z$. Considering the above-cloud component only:

$$
-\ln \left(\frac{I_{i}}{I_{c}}\right)=\int_{0}^{z} k_{\mathrm{O} 2}\left(z^{\prime}\right) \mathrm{d} z^{\prime}+\tau_{02, c}
$$

While we use the term $z$ for path here, this may also be expressed in pressure terms (i.e., in $\mathrm{hPa}$ ), in which case the extinction coefficient is in $\mathrm{hPa}^{-1}$. While $k_{\mathrm{O} 2}\left(z^{\prime}\right)$ varies through the atmosphere, for illustration we assume an effective mean extinction coefficient $<k_{\mathrm{O} 2}>$ such that $\int_{0}^{z} k_{\mathrm{O} 2}\left(z^{\prime}\right) \mathrm{d} z^{\prime} \cong<k_{\mathrm{O} 2}>\Delta z$, then we relate path to cloud top pressure $P_{\text {top }}$ and the cosine of solar zenith angle $\mu$ as $\Delta z=\left(1+\mu^{-1}\right) P_{\text {top }}$ :

$$
-\ln \left(\frac{I_{i}}{I_{c}}\right) \cong<k_{02}>\left(1+\mu^{-1}\right) P_{\text {top }}+\tau_{02, c}
$$



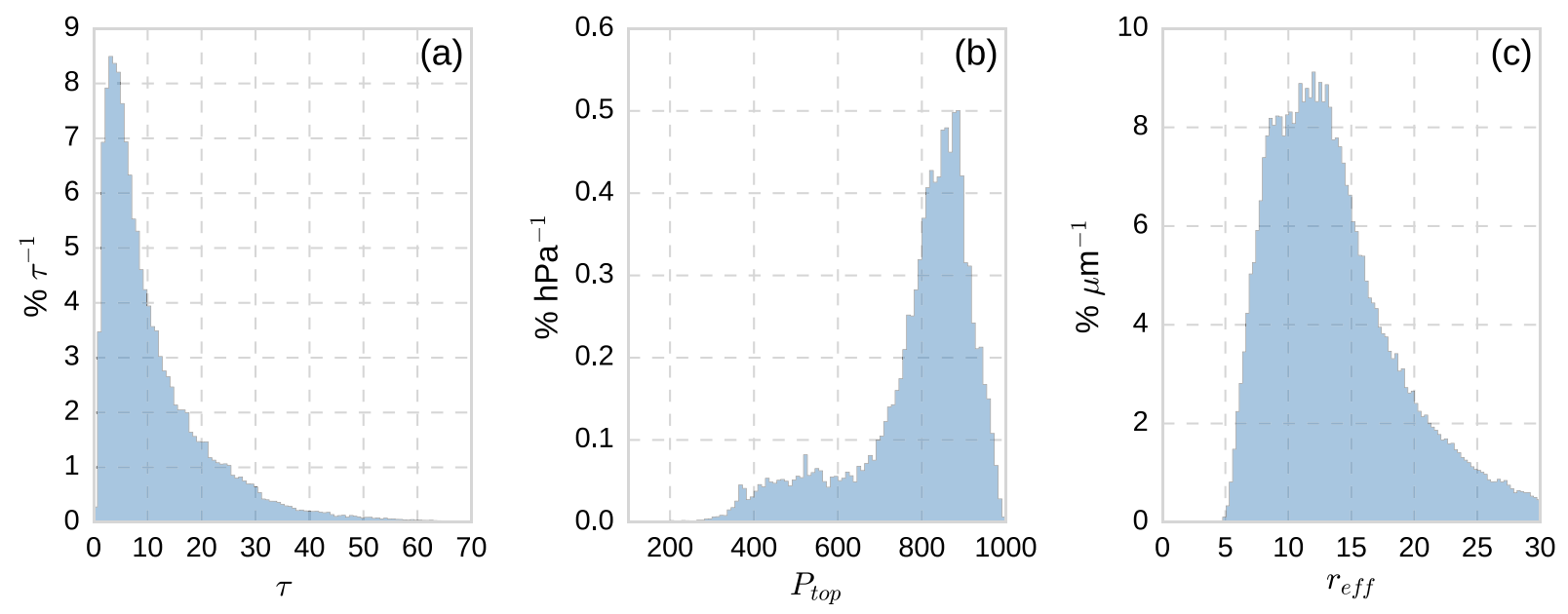

Figure 3. Histograms showing the distribution of cloud properties according to (a) MODIS optical depth $\tau$ (\% per optical depth), (b) CALIPSO $P_{\text {top }}\left(\%\right.$ hPa ${ }^{-1}$ ), and (c) MODIS effective radius $\left(\% \mu \mathrm{m}^{-1}\right)$.

For relatively small changes in $P_{\text {top }}$ where $k_{\mathrm{O} 2}\left(z^{\prime}\right)$ varies little, if we regress $\ln \left(/ / I_{c}\right)$ against above-cloud path and assume that the above-cloud and within-cloud components are uncorrelated, then the gradient of our regression will be proportional to $\left(1+\mu^{-1}\right) P_{\text {top }}$ while the intercept is related to the properties of the within-cloud optical depth distribution. In reality line broadening through the atmosphere results in a nonlinear relationship for wide ranges of $P_{\text {top. }}$. Furthermore, we assume no correlation between above- and within-cloud extinction, and any model-based retrieval will be sensitive to this. Our simulations with scaled cloud thicknesses help illustrate the sensitivity of this assumption to within-cloud path. Our $P_{\text {top }}$ look-up table is developed by binning simulated $I / I_{c}$ by the input $\Delta z$ and then entering the bin median radiance ratio and $\Delta z$ as our look-up table entry.

The performance of the look-up tables is then assessed by estimating $P_{\text {top }}$ and $\tau$ for November 2015 from the L1bSc spectra and comparing the results with observed CALIPSO $P_{\text {top }}$ and MODIS $\tau$. In addition to validation, this step also provides an upper limit on the OCO-2 retrieval uncertainty to compliment the lower bound retrieved from the precision limit calculated using equation (4), as the difference between the OCO-2 retrieved property and that from MODIS or CALIPSO will include all OCO-2-related uncertainties in addition to collocation error. As collocation error is greater than zero, the true retrieval uncertainty is smaller than this value. In addition, a future formal retrieval that makes use of individual channels and full profile information to better determine the true profile of $\mathrm{O}_{2}$ absorption coefficients will further reduce uncertainties.

\section{Results}

\subsection{Cloud Properties}

Maps of mean MODIS- and CALIPSO-observed cloud properties on a $4 \times 4^{\circ}$ latitude-longitude grid are shown in Figure 2. As the measurements occur during November and there is a limit placed on the valid solar zenith angle, the measurements reach further to the south than they do the north. There are relatively more samples in the cloudy areas of the Southern Ocean and over the South American and Namibian stratocumulus regions. Noncloudy regions or those where high clouds dominate are strongly undersampled as we exclude MODIS-identified ice clouds.

Figure 3 displays histograms of the cloud properties for the full set of simulations, demonstrating a preponderance of low clouds due to our selection of water clouds. The Pearson correlation coefficients between pairs of cloud properties are $-0.26\left(\tau-P_{\text {top }}\right), 0.19\left(\tau-r_{\text {eff }}\right),-0.18\left(r_{\text {eff }}-P_{\text {top }}\right)$. These weak correlations ( $<7 \%$ of variance explained) imply that our regression analysis of OCO-2 radiance response to cloud properties will therefore respond mostly to the property of interest rather than being driven by changes in a correlated variable. 

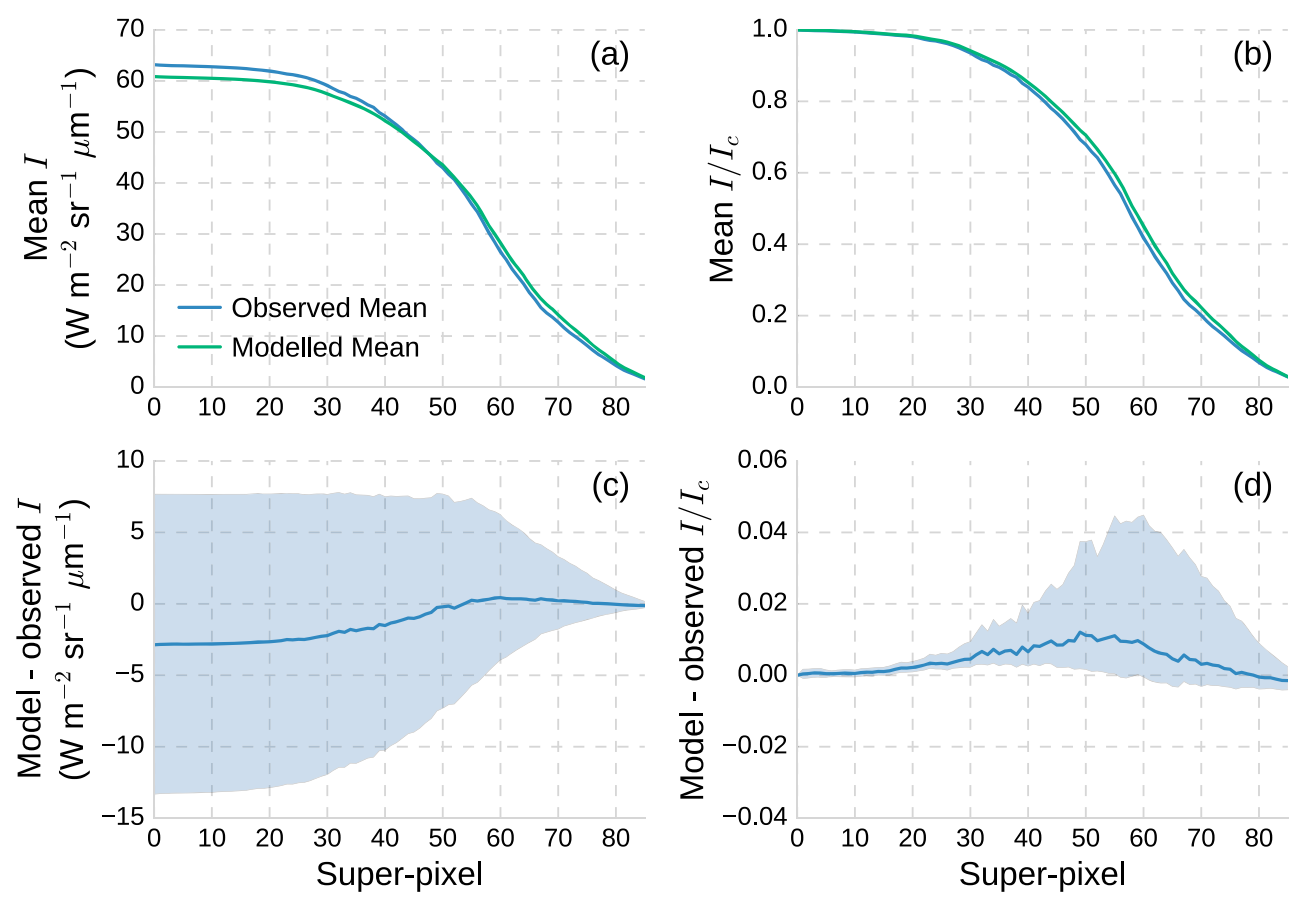

Figure 4. (a) Mean observed (blue) and simulated (green) radiances, with spectra ranked by mean channel absorption. (b) Mean observed and simulated ratio $I / I_{c}$. (c) Simulated minus observed radiance median (solid line) and interquartile range (shaded). (d) Simulated minus observed $I / I_{C}$ ratio median (solid line) and interquartile range (shaded).

\subsection{Observed and Simulated Radiances}

Figure 4 shows that mean simulated radiance is $6 \%$ lower than observations in continuum channels, with an interquartile range of $(-20,+17) \%$. This difference is due to some combination of collocation error, cloud 3-D effects, errors in the MODIS and CALIPSO retrievals, OCO-2 instrumental uncertainty, and modeling error. We expected that because we only simulate cases where MODIS retrieves a cloud, collocation error may mean that there are cases where OCO-2 measures a clear sky, thus favoring brighter simulated spectra, but this effect is outweighed in the full sample. Three-dimensional cloud effects refer to the differences between observed and simulated spectra due to the simulation assumption of a horizontally homogeneous cloud in the simulation. For example, in reality photons may leak through the sides of clouds and the effect on retrievals depends on cloud type, illumination and viewing geometry, and measurement resolution. These are known to affect retrievals and have been specifically considered for both MODIS and OCO-2 [Várnai and Marshak, 2002; Merrelli et al., 2015]. Figures S1 and S2 in the supporting information show that the continuum is unaffected when cloud pressure thickness is scaled from 0.5 to 4 , although absorption band radiances differ by $\pm 2 \%$ of the continuum radiance.

Figure 5 shows 2-D histograms comparing modeled and observed radiances in the brightest continuum Aband superpixel, including subsamples based on the variation in neighboring sounding continuum radiance. Neighboring radiances are defined as those within \pm 1 sounding along track, \pm 1 sounding across track, and all linear combinations thereof, resulting in five neighbors for soundings at the swath edge and eight for all others. We define a heterogeneity parameter by dividing the standard deviation in local $I / \mu$ by its mean and select the approximately $10 \%$ of soundings with the lowest value to represent homogeneous cloud cases (heterogeneity parameter $<0.043$ ) and the approximately $10 \%$ with the highest value to represent heterogeneous cases (heterogeneity parameter $>0.43$ ). Low spatial variance is indicative of homogeneous cases, so if model-observation differences are reduced then this indicates a contribution from collocation error. While the full-sample root-mean-square error (RMSE) is $\pm 22.4 \mathrm{~W} \mathrm{~m}^{-2} \mathrm{sr}^{-1} \mu \mathrm{m}^{-1}$ and best fit line is 1.00, selecting homogeneous-cloud cases reduces RMSE by $31 \%$ to $15.5 \mathrm{~W} \mathrm{~m}^{-2} \mathrm{sr}^{-1} \mu \mathrm{m}^{-1}$ and the best fit ratio becomes 1.09. Meanwhile, selecting heterogeneous-cloud cases increases RMSE by $50 \%$ to $33.7 \mathrm{~W} \mathrm{~m}^{-2} \mathrm{sr}^{-1} \mu \mathrm{m}^{-1}$ and a best fit ratio of 0.61 indicates a substantial increase in bias. For reference, if the error divided by the 

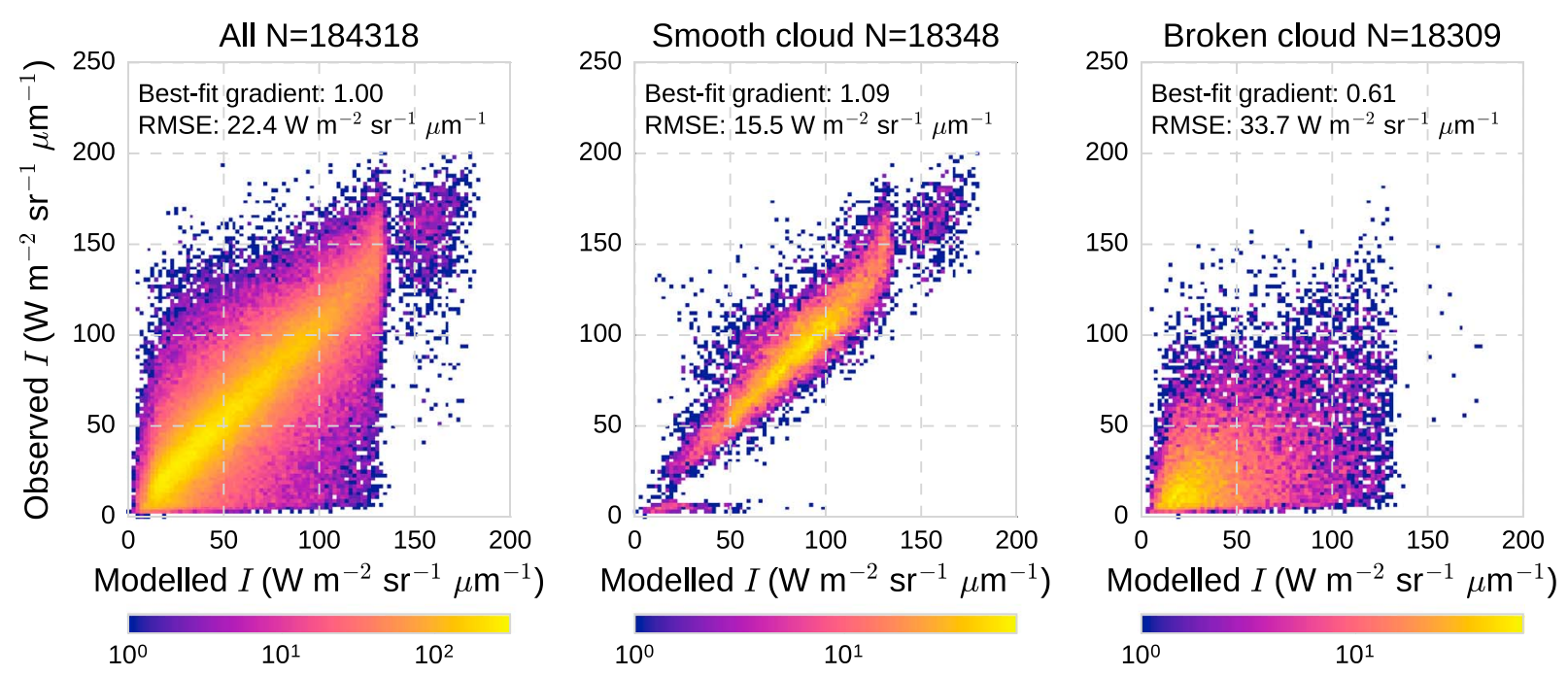

Figure 5. 2-D histograms of modeled and observed OCO-2 radiances in an A-band continuum channel. (left) All cases ( $N=184,318)$. (middle) Horizontally homogeneous cloud cases where the standard deviation of neighboring OCO-2 radiances is $<4.3 \%$ of the neighbor mean. (right) Cases where this ratio is $>43 \%$, representing horizontally heterogeneous clouds. In each case the gradient is reported for a linear fit forced through the origin, and RMSE is that of modeled minus observed with no correction for the trend bias.

mean for each sample is taken, we obtain $\pm 36.9 \%$ for all cases, $\pm 17.9 \%$ for homogeneous cases, and $\pm 69.6 \%$ for heterogeneous cases. These results are qualitatively similar to those observed in a comparison between MODIS and POLDER $\tau$ for overcast and broken-cloud scenes [Zeng et al., 2012] which were attributed largely to cloud-fraction disagreement. Cloud-fraction disagreement will also be present here as the OCO-2 radiance simulations assume that a homogeneous cloud fills the full footprint and MODIS brokencloud cases will by definition have nonuniform cloud coverage. As these are continuum radiances, cloud thickness has a near-zero effect on these results.

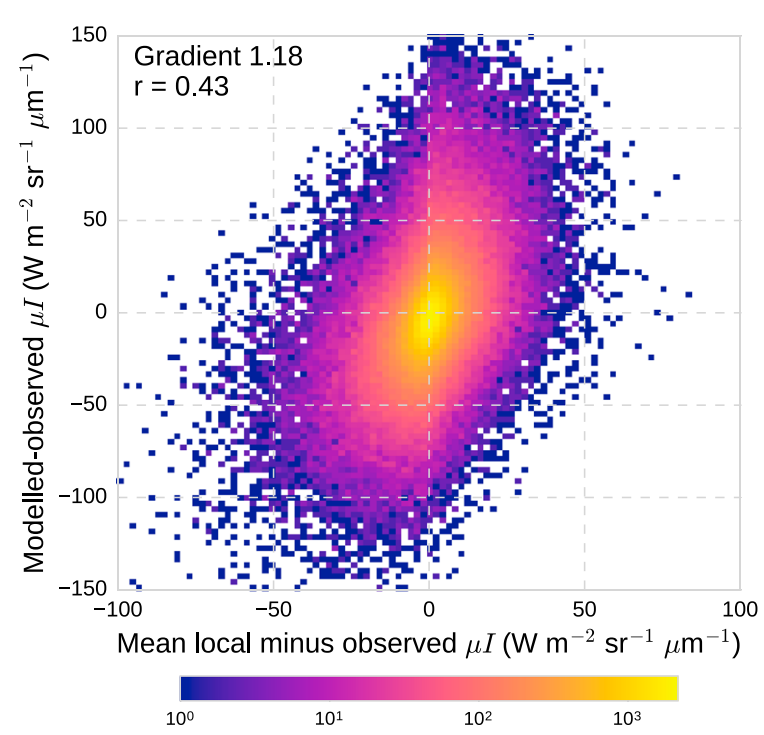

Figure 6. 2-D histogram of simulation error for the brightest continuum channel (modeled minus observed radiance, not corrected for solar zenith angle) as a function of the local radiance bias defined as the mean of neighboring soundings minus the observed value within the pixel.
Figure 6 shows that simulated values are brighter than observations when the observation sounding is surrounded by a brighter field of view (Pearson correlation coefficient, $r=0.43$ ), a pattern consistent with the presence of collocation errors.

Next we consider the response of radiances to changes in cloud properties in both models and observations. The results we present are for absolute observed radiance corrected for solar zenith angle, $l / \mu$ in response to changes in optical depth $\tau$, and for the change in the radiance ratio $s=I / I_{c}$ in response to changes in above-cloud photon path which is related to cloud top pressure using equation (8).

These results are presented for the continuum, a midabsorption case and a line-core case by selecting the 2nd, 61st, and 76th superpixels. We select the 2 nd superpixel to 

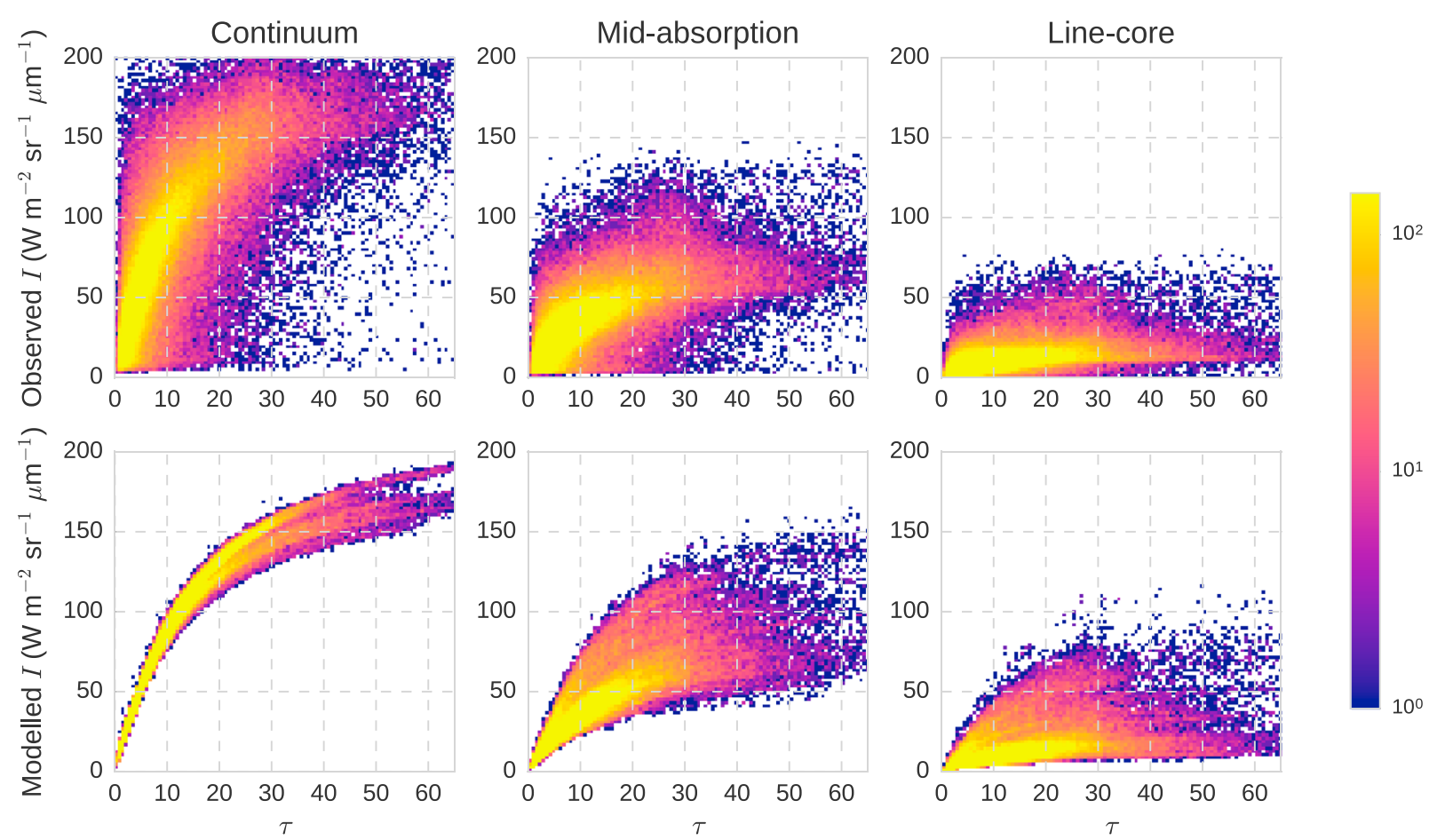

Figure 7. (top row) Observed and (bottom row) modeled radiance as a function of cloud optical depth for super pixels in the (left column) continuum (superpixel rank 2), for (middle column) midabsorption (superpixel rank 61), and for (right column) near line cores (superpixel rank 76).
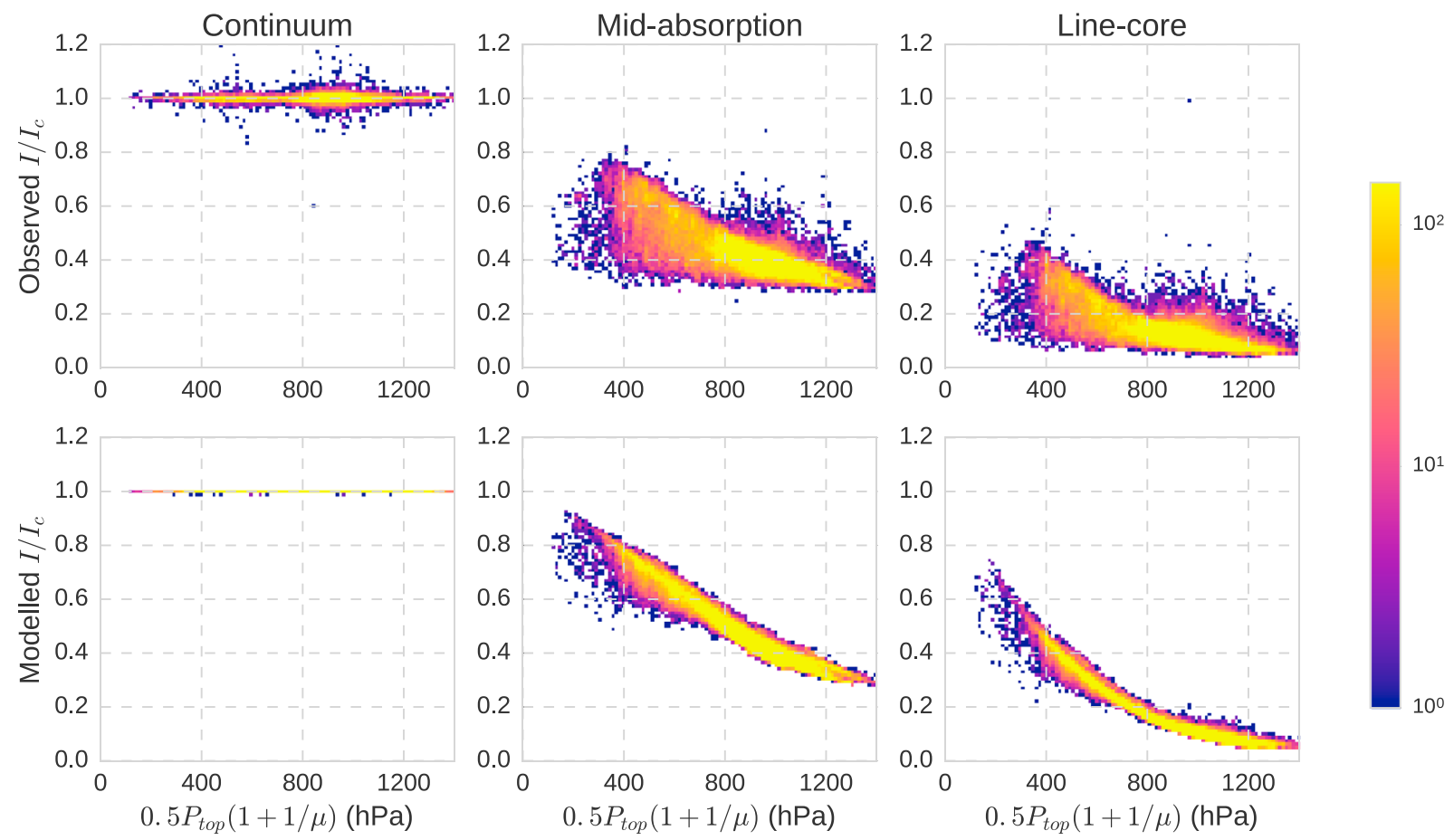

Figure 8. (top row) Observed and (bottom row) modeled histograms of the ratio of radiance $I / I_{c}$ as a function of estimated above-cloud optical path length for super pixels in the (left column) continuum (superpixel rank 2), for (middle column) midabsorption (superpixel rank 61), and for (right column) near line cores (superpixel rank 75). 

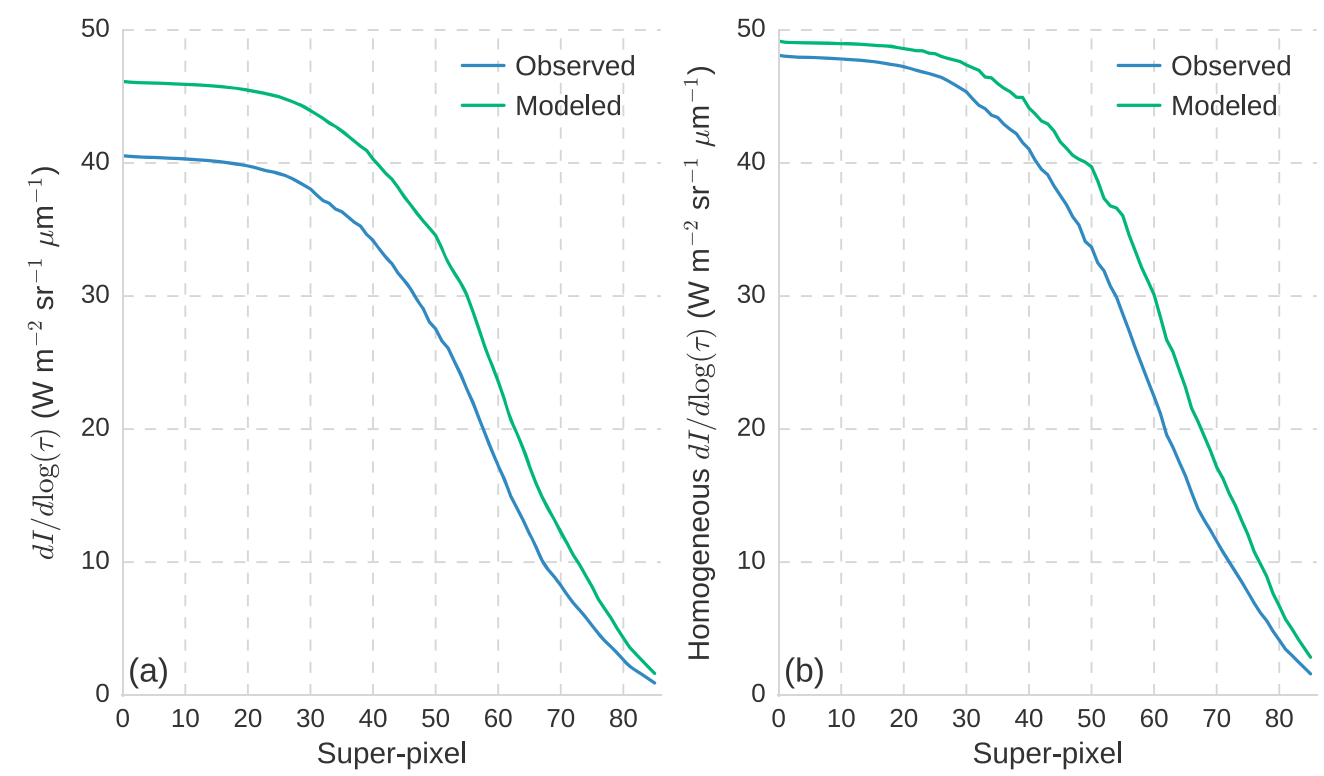

Figure 9. Regression coefficients showing the response of observed and simulated OCO-2 radiance scaled to account for solar zenith (i.e., $1 / \mu$ ) to cloud properties: (a) response to natural logarithm of optical depth in all cases; (b) response to natural logarithm of optical depth in the homogeneous cloud cases identified in Figure $5 \mathrm{~b}$.

represent the continuum as for the first superpixel the $I / I_{c}$ ratio is defined to be unity. Figure 7 displays histograms of the response of observed and modeled radiance to optical depth as determined from MODIS. The clearest response is in the continuum channels as expected, and the pattern of responses is visibly similar for all cases with a broader spread in the observations compared to the simulations.

Meanwhile, Figure 8 shows the histograms of the ratio $I / I_{c}$ in response to above-cloud optical path length showing cases where there is a low $I / I_{C}$ ratio despite a low path length being derived from CALIOP measurements. CALIOP's ground footprint is approximately $70 \mathrm{~m}$ in diameter so on average only around $1 \%$ of the OCO-2 footprint is sampled. In cases where CALIPSO identifies high cloud but the majority of the OCO-2 field of view consists of low (or no) clouds, the OCO-2-observed path length will naturally be much greater than that inferred from the CALIPSO cloud top pressure. There will also be cases where the CALIPSO lidar is attenuated but an underlying cloud contributes to the OCO-2-observed radiances, and in these cases the simulated $I / I_{c}$ will be much too great. We are unable to further test this but propose that this explains much of the spread in $I / I_{c}$ for low cloud top pressures in the observed midabsorption channel in Figure 8, especially as CALIPSO is seeing clouds with $P_{\text {top }}$ near $200 \mathrm{hPa}$, and these retrievals are unlikely to represent liquid clouds. An additional factor that may contribute is if high cloud thicknesses are biased relative to our assumed values: although Figures $\mathrm{S} 3$ and $\mathrm{S} 4$ show that when cloud pressure thickness is scaled by $0.5-4$ the changes are not sufficient to explain the full difference. Figures $\mathrm{S} 5$ and $\mathrm{S} 6$ show that selecting for homogeneous cloud cases particularly improves the continuum response to optical depth.

\subsection{Observed and Simulated Regression-Based Responses to Cloud Properties 3.3.1. Response of Measured Radiance to Cloud Optical Depth}

Using the methodology described in section 2.4 , we derive the response of $I / \mu$ or $I / I_{c}$ to MODIS and CALIPSO cloud properties for each superpixel using linear regression.

Figure 9 a shows the regression coefficients for $I / \mu$ as a function of the natural logarithm of MODIS optical depth for each superpixel, with observed response patterns similar to those simulated, but with a $18 \%$ stronger response from the radiative transfer model. Figure $S 7$ shows little sensitivity in continuum channels to the assumed cloud pressure thickness, meaning our optical depth look-up table is robust to $\Delta P$.

Figure $9 \mathrm{~b}$ focuses on the homogeneous cloud cases from Figure 5 where simulated continuum response is just $2 \%$ greater than observations. This suggests that cloud heterogeneity and collocation error explain much of the apparent difference in the full sample. In addition, the absorption band response is proportionally 

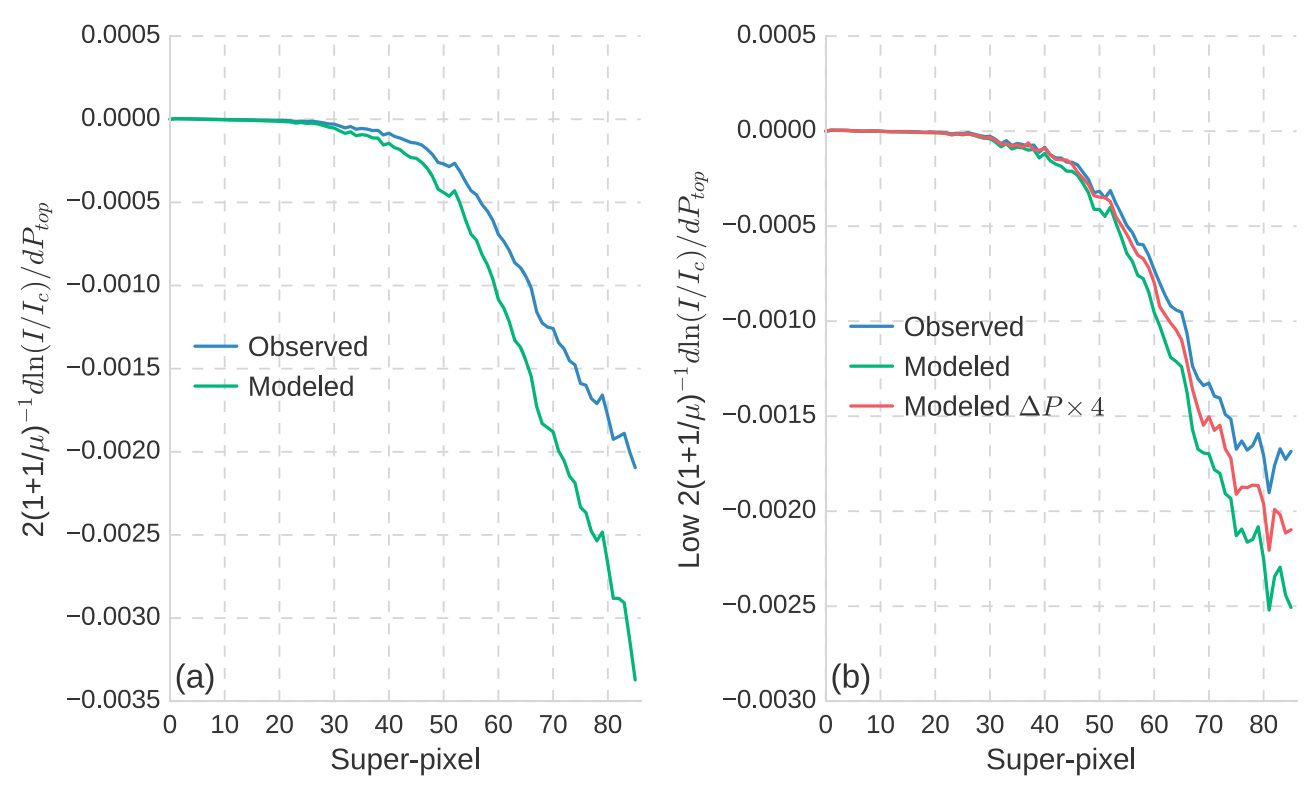

Figure 10. Response of the natural logarithm of radiance ratio $I / I_{C}$ to the above-cloud path expressed as the regression coefficient for each superpixel from observations and simulations. (a) Response for all cases; (b) response for low clouds $\left(P_{\text {top }}>680 \mathrm{hPa}\right)$. Figure $10 \mathrm{~b}$ also shows the simulated regression when cloud pressure thickness is scaled by a factor of 4 to illustrate sensitivity in this case.

much greater in simulations than observations. We propose that this is due to the simulation assuming a single cloud layer at the CALIOP pressure level, whereas in reality the OCO-2 field of view may contain lower clouds. In cases such as this the CALIOP cloud top pressure will be low, but the OCO-2 mean path length will be high, causing the wide spread in the midchannel observations at low $P_{\text {top }}$ in Figure 8 , which reduces the gradient of the regression line.

\subsubsection{Response of Radiance Ratio}

Figure 10 shows the response of the natural logarithm of the radiance ratio $I / I_{c}$ to the estimated above-cloud path, following equation (8) that states that these two values are proportional given the approximation of a near-uniform vertical profile of $\mathrm{O}_{2}$ extinction coefficient. Figure 10a shows that the model $\ln \left(I / I_{c}\right)$ responds more strongly than the observed values which follows from inspection of the midabsorption panels of Figure 8. CALIOP identifies some very high clouds where OCO-2 measures $I / I_{c}$ consistent with lower clouds, which reduces the calculated gradient in $1 / I_{c}$ as a function of above-cloud path and matches our previous discussion regarding collocation and layering issues with CALIOP $P_{\text {top. }}$. This is supported by Figure $10 \mathrm{~b}$ which shows the regression results from low clouds only $\left(P_{\text {top }}<680 \mathrm{hPa}\right)$, in which case the model-observation difference is smaller. It is further reduced when simulated cloud pressure thickness is larger, shown in the red line of Figure $5 \mathrm{~b}$. Figures $\mathrm{S} 8$ and $\mathrm{S} 9$ show further pressure thickness comparisons.

The improved agreement in the response of $I / I_{c}$ to above-cloud path $\Delta z$ when thicker clouds are simulated means that the change in $\tau_{\mathrm{O} 2}$ in response to $\Delta z_{c}$ is likely misrepresented. This may be due to a bias in our assumed cloud pressure thickness that correlates with cloud top pressure, but given the weak correlation between $\Delta z$ and other cloud properties, we favor an alternative explanation. We propose that our approximation following equation (7) is invalid, namely, that $\left.\int k_{\mathrm{O} 2}\left(z^{\prime}\right) d z^{\prime} \neq<k_{\mathrm{O} 2}\right\rangle \Delta z$. A thicker simulated cloud pressure thickness increases simulated $k_{\mathrm{O} 2}$ within the cloud, with greater enhancements for lower clouds. The simulations with thicker clouds show responses closer to those observed, and while this may be partly because the real-world clouds are thicker, it may also be a representation of errors in the interpolated ECMWF profiles that do not adequately represent within-cloud conditions.

\subsubsection{Implied Single-Sounding Precision From Observed Sensitivities}

The theoretical precision of a measurement may be expressed in terms of the change in the observed property that would lead to a measured response of $1 \sigma$, where $\sigma$ is the measurement precision. The theoretical limit of precision may be obtained for each property by taking the instrumental radiance uncertainties 

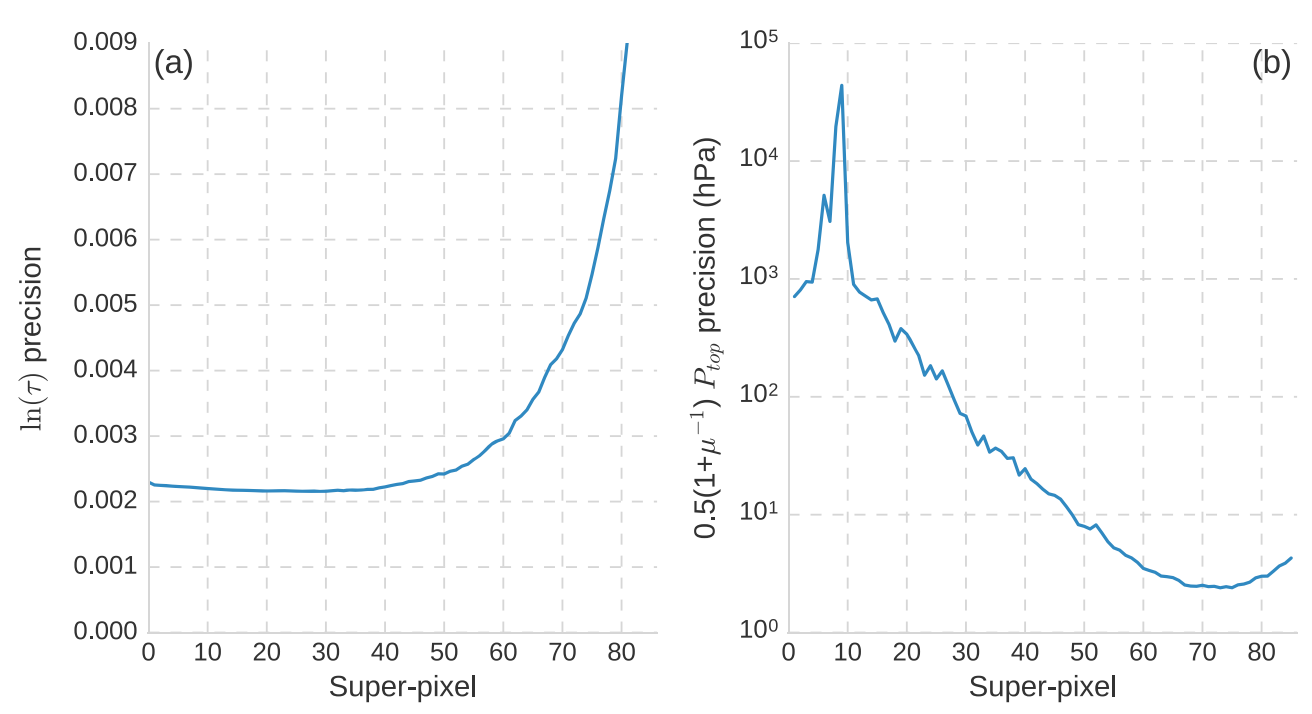

Figure 11. Theoretical limiting precision based on the mean $\mathrm{L} 1 \mathrm{bSc}$ instrumental uncertainties combined with the relevant sensitivities, which are the observed regression coefficients shown in Figures 9 and 10 for changes in (a) MODIS $\ln (\tau)$ and (b) CALIOP above-cloud path based on $P_{\text {top }}$. The best precision is $\pm 0.002 \operatorname{in} \ln (\tau)$, or approximately $\pm 0.2 \%$ in $\tau$, and $\pm 2.4 \mathrm{hPa}$ in above-cloud path.

reported with the $\mathrm{L} 1 \mathrm{bSc}$ product and dividing them by the sensitivities derived in sections 3.3.1 and 3.3.2. The measurement precision for $\ln (\tau)$, which we label $\delta \ln (\tau)$, from a given superpixel with measurement uncertainty $\sigma_{l}$, for example, is

$$
\delta \ln (\tau)=\left(\frac{\partial l}{\partial \ln (\tau)}\right)^{-1} \sigma_{l}
$$

We approximate the partial derivative with the regression coefficients from Figure 9, while $\sigma_{l}$ is taken to be the mean reported radiance error in that channel across all soundings, which is equivalent to assuming that the channels within each superpixel have perfectly correlated errors. Similarly, we estimate the precision in one-way above-cloud path as $0.5\left(1+\mu^{-1}\right) \delta P_{\text {top }}=\Delta z / 2$ from

$$
\delta \Delta z=\left(\frac{\partial \ln \left(\frac{l}{l_{c}}\right)}{\partial \Delta z_{p}}\right)^{-1} \sigma_{\ln \left(\frac{l}{l_{c}}\right)}
$$

where the partial derivative is approximated with the regression coefficients from Figure 10 . The $\ln \left(/ / I_{c}\right)$ uncertainty is determined by quadrature assuming uncorrelated uncertainty:

$$
\sigma_{\ln \left(\frac{I}{I_{c}}\right)}=\sqrt{\left(\frac{\sigma_{I}}{l}\right)^{2}+\left(\frac{\sigma_{I_{c}}}{I_{c}}\right)^{2}}
$$

The results of equations (9) and (10) are shown in Figure 11, with a best possible $\ln (\tau)$ precision of \pm 0.002 (approximately $0.2 \%$ in absolute $\tau$ ) and $\Delta z_{p}$ precision of $\pm 2.4 \mathrm{hPa}$. Most of the information about optical depth is obtained in the continuum, while cloud top pressure requires measurements within the absorption bands, although precision decreases in the line cores when too much of the signal has been absorbed.

\subsection{Development and Validation of Look-Up Table-Based Retrieval}

As described in section 2.5 we produce look-up tables relating solar-zenith-corrected radiance $I$ to $\ln (\tau)$ and radiance ratio $I / I_{c}$ to above-cloud path using the simulated spectra binned by cloud property. A look-up table 

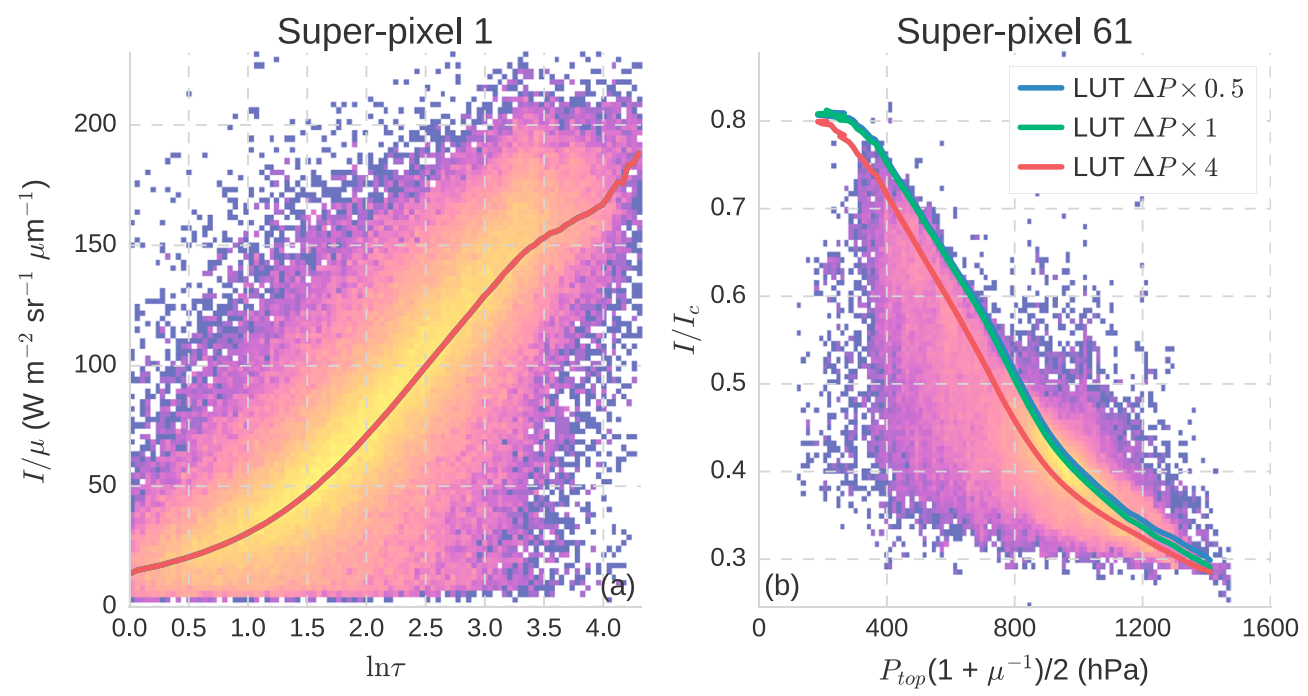

Figure 12. Example look-up tables developed from the radiative transfer simulations, (a) using absolute radiance corrected for solar zenith angle in the 1st superpixel as a function of the natural logarithm of MODIS optical depth; (b) for radiance ratio as a function of above-cloud path for the 61st superpixel. In each case the relevant observation-based 2-D histogram is shown in the background. In each case the colored lines represent the derived look-up tables from the set of simulations in which the cloud pressure thickness is scaled from 0.5 to 4.0 as labeled in the caption.

relating the measurement to the cloud property of interest is generated for each superpixel, and then this table is used with the observed OCO-2 radiances to derive estimates of $\tau$ and $P_{\text {top }}$ from each superpixel independently. This process is illustrated in Figure 12, which shows example look-up tables for optical depth using the first superpixel and for above-cloud path using the 61st superpixel. Derived look-up tables are shown for each of the cloud pressure thickness assumptions, with scale factors from 0.5 to 4.0. The $\ln (\tau)$ example look-up table is not responsive to cloud thickness, although the $P_{\text {top }}$ look-up table is sensitive to this choice.
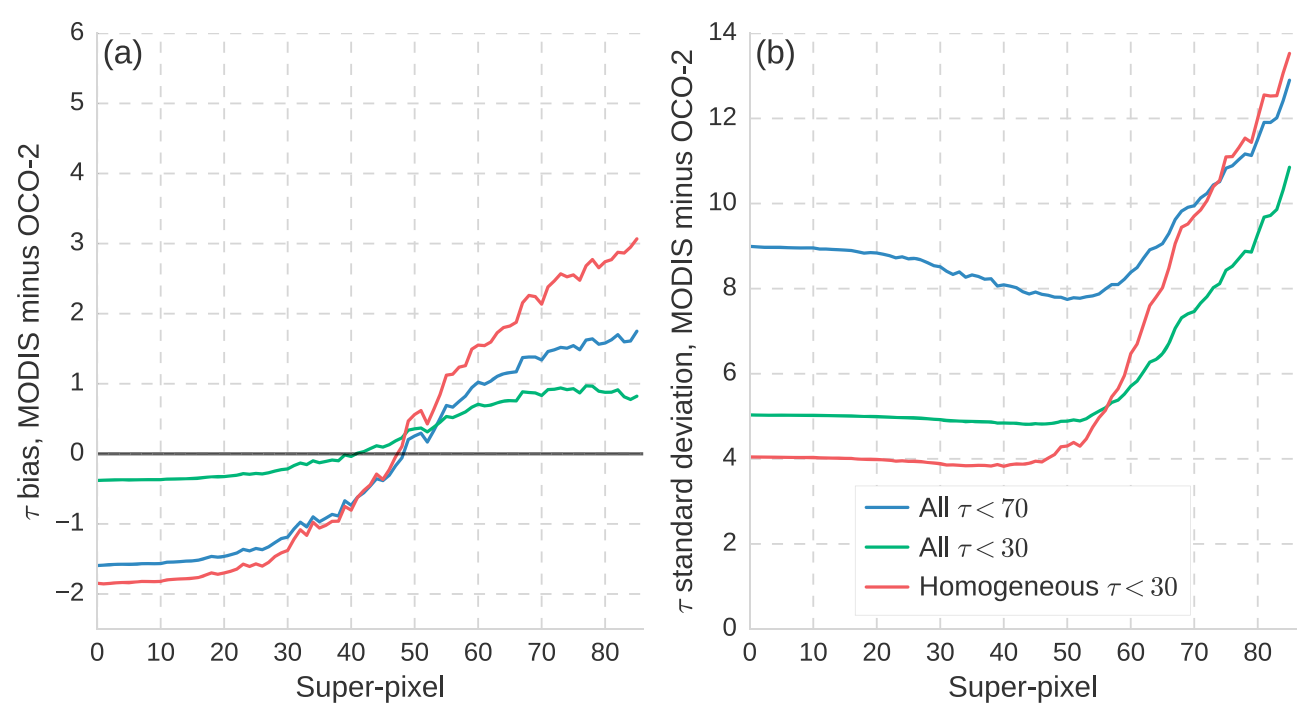

Figure 13. Comparison of MODIS optical depth and the calculated value using a single OCO-2 super pixel at a time. (a) Bias in predicted optical depth in November 2015 reported as mean MODIS $\tau$ minus retrieved OCO- 2 . (b) Standard deviation of MODIS $\tau$ minus that calculated from OCO-2. 

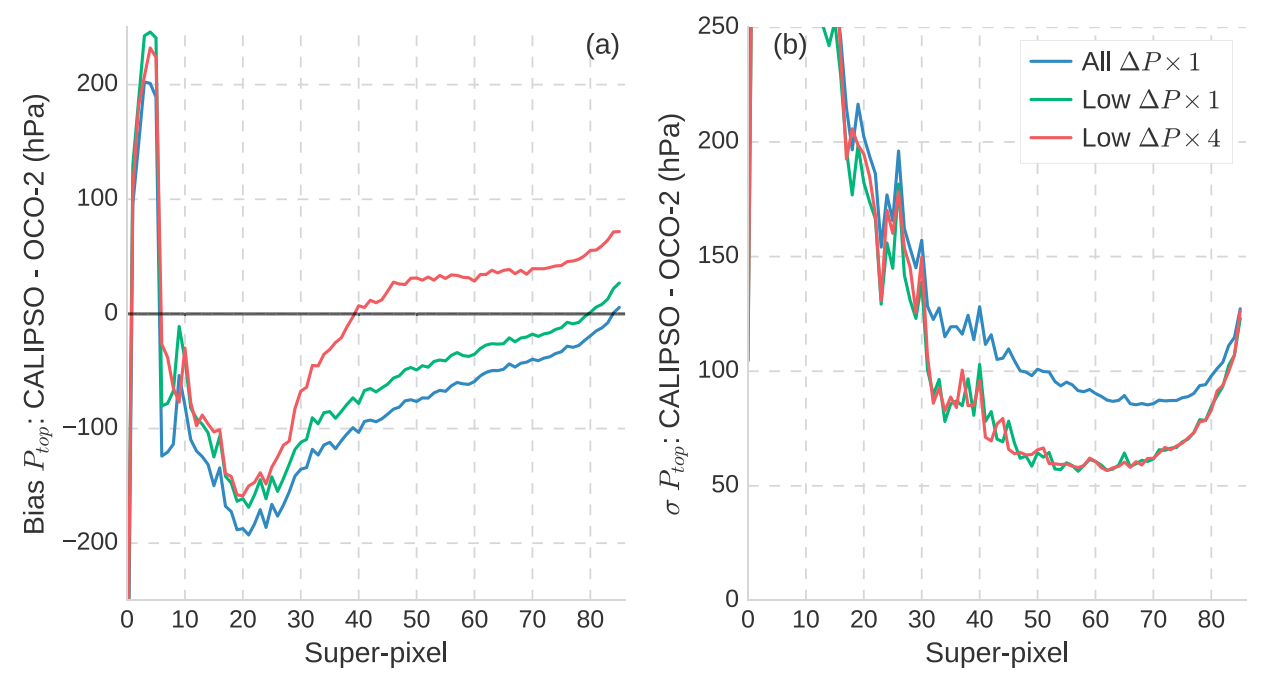

Figure 14. Comparison of CALIPSO cloud top pressure and that calculated using a look-up table with a single OCO-2 super pixel at a time for November 2015 (a) bias as CALIOP minus OCO-2 and (b) standard deviation of these differences. All cases with the standard OCO-2 look-up table are shown in blue, for low clouds (CALIOP $P_{\text {top }}>680 \mathrm{hPa}$ ) in green, and low clouds using the look-up table developed from simulations where cloud pressure thickness is scaled by 4 in red.

We calculate these look-up tables for each superpixel and then estimate the $\tau$ and $P_{\text {top }}$ from OCO-2 L1bSc spectra, and next show the bias and standard deviation relative to MODIS or CALIPSO for a single retrieval using each super pixel separately.

Figure 13 shows the mean and standard deviation of the differences between the OCO-2 and MODIS $\tau$ values. We present the results of the retrieval where MODIS and OCO- $2<70$ to reduce the effect of cases where the retrievals are no longer strongly sensitive, and also for $\tau<30$ and the homogeneous cloud cases from Figure 5. Figure S10 shows that the bias depends on cloud optical depth; it averages to 0.07 for $5<\tau<15$ but grows in magnitude for thicker clouds. Standard deviation is smallest in the continuum bands, where it is reduced for $\tau<30$ or homogeneous clouds. The true retrieval error is likely smaller than the error reported here as it is not subject to collocation error.

For cloud top pressure, the mean bias and standard deviation of OCO-2 retrievals relative to CALIPSO are shown in Figure 14. The difference is very large in the continuum channels, which contain almost no photon path information, but shrinks in the absorption bands. Selecting for low clouds (CALIOP $P_{\text {top }}>680 \mathrm{hPa}$ ) reduces both bias and standard deviation as expected. The results when the look-up tables are produced from simulations with the standard cloud pressure thickness or with a scaling of 4 are also shown: this has little effect on the standard deviation but substantially changes the bias. For the 61st superpixel, the CALIOP-OCO-2 bias is $-35.2 \mathrm{hPa}$ for the standard cloud pressure thickness, or $+28.6 \mathrm{~Pa}$ when $\Delta P$ is scaled by 4 . The standard deviation is $\pm 60.7 \mathrm{hPa}$ in each case. We also produced a look-up table by randomly sampling from the $\Delta P \times 1$ or $\Delta P \times 4$ simulations for each sounding, and for the 61 st superpixel this results in a bias of $-5.0 \mathrm{hPa}$ and a standard deviation of $\pm 60.9 \mathrm{hPa}$.

Our cloud pressure thickness assumptions therefore have greater effect on the bias in our $P_{\text {top }}$ look-up table retrieval but little effect on the variance. Once again, our reported standard deviation includes all variance introduced by differences in the OCO-2 and CALIPSO collocation. In addition, although our use of superpixels mitigates errors introduced due to different path-weighted $\mathrm{O}_{2}$ absorption coefficients, there will be errors introduced by changing meteorological conditions. A formal retrieval would include information on these conditions and how they affect $k_{\mathrm{O} 2}$, and this $\pm 60.7 \mathrm{hPa}$ standard deviation is therefore an upper limit on retrieval error.

\section{Discussion}

This paper provides the first assessment of the response of OCO-2's very high spectral resolution spaceborne A-band instrument to cloud properties using measurements from November 2015. The results shown here 
validate the A-band continuum performance of the OCO-2 L2RTM in cloudy atmospheres when the clouds are homogeneous. In addition, this work demonstrates that the derived photon path lengths respond as expected to cloud top pressure for low clouds identified from CALIOP.

Based on simulated radiances using MODIS optical depth as an input and selecting for the $10 \%$ most horizontally homogeneous scenes, simulated radiances in the continuum have a single-sounding radiance error of approximately $\pm 18 \%$. This estimate includes any uncertainty in the MODIS optical depth retrieval, suggesting smaller true model error.

Heterogeneous scenes show greater errors in terms of optical depth, but this has no distinguishable effect on the photon path length statistics and therefore does not strongly affect cloud top-height retrievals, consistent with results obtained for 3-D Monte Carlo simulations where surface albedo is low [Kokhanovsky et al., 2007]. At nadir over oceans, albedo is low, but over brighter surfaces the surface return should be considered. In cases of substantially broken cloud, photon-path length statistics could be affected by the surface contribution.

Another limitation of this analysis is the focus on single-layer clouds, assuming accurate identification by MODIS and CALIPSO. A-band radiances are sensitive to the presence of multiple cloud layers, although by exploring the implied variance of the photon-path length distribution it may be possible to identify multilayer cases [Min et al., 2004]. Where CALIOP retrieves high clouds, there is substantial variation in the values inferred from OCO-2 photon path lengths. We do not quantitatively attribute this, although possible contributing factors are multiple layers and collocation errors related to the smaller CALIOP footprint sampling a higher cloud when a larger amount of the OCO-2-measured radiance comes from lower clouds within the field of view.

In general, collocation error is expected to favor brighter modeled scenes as simulations were only performed when MODIS and CALIOP both reported a cloud, whereas the corresponding OCO-2 observation may not be of a cloudy scene. For OCO-2 glint observations over water MODIS reports a cloud that is not detected by the OCO-2 cloud filter in 8.5-10.6\% of cases depending on time of year [Taylor et al., 2016], suggesting that a number of our MODIS-determined cloudy scenes will not appear cloudy to OCO-2.

Figure 9 demonstrates that, as expected, modeled response of OCO-2 radiances to optical depth is greater than that seen in observations with better agreement when homogeneous cloud cases are selected. This supports the hypothesis of issues related to collocation error. The results presented in Figure 10 showed that observed and modeled OCO-2 responses to low-cloud $P_{\text {top }}$ are similar.

The observed OCO-2 radiance responses to optical depth and cloud top pressure were then used to estimate the theoretical single-channel $(\tau)$ or double-channel $\left(P_{\text {top }}\right)$ retrieval precision based only on instrumental uncertainty. The derived limits were \pm 0.002 in $\ln (\tau)$ and $\pm 2.4 \mathrm{hPa}$ in above-cloud path based on regressions: a formal retrieval would exploit additional nonlinearities of these sensitivities.

The reported instrumental-precision uncertainties are unrealistically low for real-world applications. A complementary error estimate was developed by applying simple single- or double-channel retrievals of $\tau$ and $P_{\text {top. }}$. Figure 14 demonstrates that for cases where CALIOP retrieves low clouds ( $P_{\text {top }}>680 \mathrm{hPa}$ ), a simple look-up table-based retrieval using the OCO-2 photon path lengths from two superpixels results in an OCO-2-estimated $P_{\text {top }}$ with bias that depends on the assumed cloud thickness and a standard deviation relative to CALIOP of $\sigma \sim 60 \mathrm{hPa}$. Comparison of single-channel $\tau$ estimated from OCO- 2 with the value retrieved from MODIS results in a large standard deviation of $\sim 9$, although this falls to $\sim 5$ for clouds of optical depth $<30$. Much of this is likely due to spatial variability and collocation errors.

Our dual-channel $P_{\text {top }}$ precision is similar to that reported for other instruments such as MERIS, although our comparison with CALIOP shows a standard deviation that is larger than the reported root-mean-square error (RMSE) for airborne-lidar-based validation of the MERIS retrievals (bias $22 \mathrm{hPa}$, RMSE $24 \mathrm{hPa}$ ) [Lindstrot et al., 2006]. By scaling cloud pressure thickness we may obtain a lower bias, however.

Our future retrieval will use these look-up tables to produce prior estimates of the initial cloud top pressure and optical depth and will then use an optimal estimation scheme with more channels and without the superpixel smoothing. This will also only be performed where the OCO-2 sounding is identified as cloudy, avoiding cases in this data set where OCO-2 is likely not seeing clouds identified by MODIS and CALIOP. An optimal estimation scheme using a greater number of channels will exploit sensitivities at different 
layers of the atmosphere and to obtain more information about photon path length statistics. For example, channels near the line core are more responsive to high clouds, whereas less-absorbing channels are required for low clouds as otherwise above-cloud absorption removes the radiance. The atmospheric state can also affect the response of individual channels, with warmer and moister atmospheres changing the $\mathrm{O}_{2}$ absorption in a given channel. In addition, a combination of multiple scattering and differential line broadening within the cloud relative to above the cloud should allow disaggregation of photon path length into an above-cloud and within-cloud component. These responses occur in the OCO-2 L2RTM, but the responses are more complex and the superpixel approach adopted in this paper reduces the sensitivity of radiances to the cloud pressure thickness; hence, we avoid an in-depth analysis here.

The work presented here has developed simple retrievals for cloud optical depth and cloud top pressure which will be used to provide prior information necessary for a more detailed retrieval.

\section{Conclusions}

A number of A-band sensors are currently available, but the majority of them have wide instrument line shapes and so are unable to sample a wide range of effective oxygen absorption coefficients in the A-band, which fundamentally limits their ability to extract information about photon path length and therefore cloud properties. OCO-2 and GOSAT both offer much higher spectral resolution and while GOSAT retrieves more polarimetric information that is informative of clouds [Sanghavi et al., 2015], it has a much larger footprint than OCO-2 (total area $>20$ times greater), meaning that cloud heterogeneity would be a greater issue. Additionally, we have been able to directly exploit OCO-2's formation flying with Aqua and CALIPSO which provide cloud optical properties with their MODIS and CALIOP instruments, respectively.

The MODIS imager provides cloud-optical depth and effective radius, while the CALIOP lidar retrieves cloud top pressure. OCO-2 allows a joint retrieval of both, which CALIOP cannot do in optically thick clouds due to lidar attenuation. MODIS does such a retrieval, but by using infrared emission to determine cloud height it requires corrections over low clouds with complex above-cloud temperature profiles, such as the inversions common over marine stratocumulus. OCO-2 retrievals based on reflected photon path length statistics do not require such a correction and so are less sensitive to errors introduced by temperature inversions.

Here we have used collocated MODIS-CALIOP-OCO-2 data from November 2015 to confirm an adequate implementation of the OCO-2 L2RTM for simulating cloudy scenes when MODIS and CALIPSO identify single-layer liquid clouds over ocean. Three-dimensional cloud radiative effects can be identified from homogeneity between nearby OCO-2 soundings, and simulations of continuum radiance in homogeneous cloud cases show an individual sounding standard deviation of $\pm 18 \%$, which includes residual MODIS errors suggesting that true model error is smaller. The observed radiance response to optical depth from MODIS agrees well with simulations, as do inferred photon-path lengths from OCO-2 observations in response to cloud top pressure for low clouds identified by CALIOP. CALIOP-identified high clouds show bias and a much greater spread which may be related to cloud layers hidden from CALIOP due to attenuation, or horizontal heterogeneity as the OCO-2 footprint is much larger than that seen by CALIOP.

Based on the cloud responses, we estimate an instrumental precision limit of $\pm 0.002 \mathrm{in} \ln (\tau)$ and $\pm 2.4 \mathrm{hPa}$ in above-cloud path for a simple joint retrieval based on a single superpixel (optical depth) or two superpixels (cloud top pressure). When this model-developed retrieval is applied to the observed November 2015 data, standard deviation in optical depth retrieval error is \pm 9.0 and in cloud top pressure is $\pm 61 \mathrm{hPa}$ against MODIS and CALIOP values, respectively. These errors are likely overstated for the true state seen by OCO-2, as all MODIS, CALIOP, and collocation errors are included in these values.

A formal retrieval is under development for liquid clouds over ocean with the aim of retrieving the pressure thickness of marine boundary layer clouds to improve physical parameterizations in climate models. Improved simulation of these clouds is important for model energy budgets and climate response. Further work will be necessary to determine the performance in glint view, over land, and for ice clouds and to use combined CloudSat-CALIPSO data to determine OCO-2's capacity to measure cloud layering. We have demonstrated that OCO-2 A-band spectra respond largely as expected to cloud properties using a combination of simulated radiances and observations. These results are promising for the future retrieval of moredetailed cloud properties using OCO-2 A-band radiances, and this analysis would not have been possible without the multisensor applications possible due to formation flying of A-Train satellites. 


\section{Acknowledgments}

The research described in this paper was performed at the Jet Propulsion Laboratory, California Institute of Technology, sponsored by NASA. M.R. is funded by the CloudSat and OCO-2 projects. Collocated data provided by Cooperative Institute for Research in the Atmosphere. The CSU/CIRA contribution to this work was supported by JPL subcontract 1439002. M.R. would like to thank Annmarie Eldering, Mike Gunson, Vivienne Payne, David Crisp, Vijay Natraj, and Chris O'Dell for helpful discussions and support. The L1bSc OCO-2 radiances are available online from the NASA Goddard GES DISC at https://disc. gsfc.nasa.gov/datacollection/OCO2 L1B_Science_7.html. The supporting information contains a. csv file listing the collocated MODIS and CALIOP cloud properties along with the information necessary to identify the OCO-2 L1bSc file and sounding.

\section{References}

Baum, B. A., W. P. Menzel, R. A. Frey, D. C. Tobin, R. E. Holz, S. A. Ackerman, A. K. Heidinger, and P. Yang (2012), MODIS cloud-top property refinements for Collection 6, J. Appl. Meteorol. Climatol., 51(6), 1145-1163, doi:10.1175/JAMC-D-11-0203.1.

Boesch, H., et al. (2015), Orbiting Carbon Observatory (OCO)-2 Level 2 Full Physics Algorithm Theoretical Basis Document, NASA Jet Propul. Lab., Calif. Inst. of Technol., Pasadena, Calif.

Bony, S., and J.-L. Dufresne (2005), Marine boundary layer clouds at the heart of tropical cloud feedback uncertainties in climate models, Geophys. Res. Lett., 32, L20806, doi:10.1029/2005GL023851.

Borg, L. A., and R. Bennartz (2007), Vertical structure of stratiform marine boundary layer clouds and its impact on cloud albedo, Geophys. Res. Lett., 34, L05807, doi:10.1029/2006GL028713.

Brenguier, J. (1991), Parameterization of the condensation process: A theoretical approach, J. Atmos. Sci., 48(2), 264-282, doi:10.1175/ 1520-0469(1991)048<0264:POTCPA > 2.0.CO;2.

Brenguier, J., H. Pawlowska, L. Schüller, R. Preusker, J. Fischer, and Y. Fouquart (2000), Radiative properties of boundary layer clouds: Droplet effective radius versus number concentration, J. Atmos. Sci., 57(6), 803-821, doi:10.1175/1520-0469(2000)057<0803:RPOBLC >2.0.CO;2.

Brient, F., T. Schneider, Z. Tan, S. Bony, X. Qu, and A. Hall (2016), Shallowness of tropical low clouds as a predictor of climate models' response to warming, Clim. Dyn., 47(1-2), 433-449, doi:10.1007/s00382-015-2846-0.

Crisp, D. (2008), NASA Orbiting Carbon Observatory: Measuring the column averaged carbon dioxide mole fraction from space, J. Appl. Remote. Sens., 2(1), 1-14, doi:10.1117/1.2898457.

Crisp, D., et al. (2016), The on-orbit performance of the Orbiting Carbon Observatory-2 (OCO-2) instrument and its radiometrically calibrated products, Atmos. Meas. Tech., 10, 59-81, doi:10.5194/amt-10-59-2017.

Drouin, B. J., et al. (2017), Multispectrum analysis of the oxygen A-band, J. Quant. Spectrosc. Radiat. Transfer, 186, 118-138, doi:10.1016/ j.jqsrt.2016.03.037.

Eldering, A., et al. (2016), The Orbiting Carbon Observatory-2: First 18 months of science data products, Atmos. Meas. Tech., 10, 549-563, doi:10.5194/amt-10-549-2017.

Ferlay, N., F. Thieuleux, C. Cornet, A. B. Davis, P. Dubuisson, F. Ducos, F. Parol, J. Riédi, and C. Vanbauce (2010), Toward new inferences about cloud structures from multidirectional measurements in the oxygen A band: Middle-of-cloud pressure and cloud geometrical thickness from POLDER-3/PARASOL, J. Appl. Meteorol. Climatol., 49(12), 2492-2507, doi:10.1175/2010JAMC2550.1.

Fischer, J., and H. Grassl (1991), Detection of cloud-top height from backscattered radiances within the oxygen A band. Part 1: Theoretical study, J. Appl. Meteorol., 30(9), 1245-1259, doi:10.1175/1520-0450(1991)030<1245:DOCTHF>2.0.CO;2.

Flatau, P. J., and G. L. Stephens (1988), On the fundamental solution of the radiative transfer equation, J. Geophys. Res., 93(D9), 11,037-11,050, doi:10.1029/JD093iD09p11037.

Frankenberg, C., C. O’Dell, J. Berry, L. Guanter, J. Joiner, P. Köhler, R. Pollock, and T. E. Taylor (2014), Prospects for chlorophyll fluorescence remote sensing from the Orbiting Carbon Observatory-2, Remote Sens. Environ., 147, 1-12, doi:10.1016/j.rse.2014.02.007.

Holz, R. E., S. A. Ackerman, F. W. Nagle, R. Frey, S. Dutcher, R. E. Kuehn, M. A. Vaughan, and B. Baum (2008), Global Moderate Resolution Imaging Spectroradiometer (MODIS) cloud detection and height evaluation using CALIOP, J. Geophys. Res., 113, D00A19, doi:10.1029/ 2008JD009837.

Huang, Y., S. T. Siems, M. J. Manton, L. B. Hande, and J. M. Haynes (2012), The structure of low-altitude clouds over the Southern Ocean as seen by CloudSat, J. Clim., 25(7), 2535-2546, doi:10.1175/JCLI-D-11-00131.1.

Koelemeijer, R. B. A., P. Stammes, J. W. Hovenier, and J. F. de Haan (2001), A fast method for retrieval of cloud parameters using oxygen A band measurements from the Global Ozone Monitoring Experiment, J. Geophys. Res., 106(D4), 3475-3490, doi:10.1029/2000JD900657.

Kokhanovsky, A. A., B. Mayer, V. V. Rozanov, K. Wapler, J. P. Burrows, and U. Schumann (2007), The influence of broken cloudiness on cloud top height retrievals using nadir observations of backscattered solar radiation in the oxygen A-band, J. Quant. Spectrosc. Radiat. Transfer, 103(3), 460-477, doi:10.1016/j.jqsrt.2006.06.003.

Kuze, A., H. Suto, M. Nakajima, and T. Hamazaki (2009), Thermal and near infrared sensor for carbon observation Fourier-transform spectrometer on the Greenhouse Gases Observing Satellite for greenhouse gases monitoring, Appl. Opt., 48(35), 6716-6733, doi:10.1364/ AO.48.006716.

Lindstrot, R., R. Preusker, T. Ruhtz, B. Heese, M. Wiegner, C. Lindemann, and J. Fischer (2006), Validation of MERIS cloud-top pressure using airborne lidar measurements, J. Appl. Meteorol. Climatol., 45(12), 1612-1621, doi:10.1175/JAM2436.1.

Merrelli, A., R. Bennartz, C. W. O'Dell, and T. E. Taylor (2015), Estimating bias in the OCO-2 retrieval algorithm caused by 3-D radiation scattering from unresolved boundary layer clouds, Atmos. Meas. Tech., 8(4), 1641-1656, doi:10.5194/amt-8-1641-2015.

Min, Q.-L., L. C. Harrison, P. Kiedron, J. Berndt, and E. Joseph (2004), A high-resolution oxygen A-band and water vapor band spectrometer J. Geophys. Res., 109, D02202, doi:10.1029/2003JD003540.

Natraj, V., and R. J. D. Spurr (2007), A fast linearized pseudo-spherical two orders of scattering model to account for polarization in vertically inhomogeneous scattering-absorbing media, J. Quant. Spectrosc. Radiat. Transfer, 107(2), 263-293, doi:10.1016/j.jqsrt.2007.02.011.

O'Brien, D. M., and R. M. Mitchell (1992), Error estimates for retrieval of cloud-top pressure using absorption in the A band of oxygen, J. Appl. Meteorol., 31(10), 1179-1192, doi:10.1175/1520-0450(1992)031<1179:EEFROC >2.0.CO;2.

O'Dell, C. W. (2010), Acceleration of multiple-scattering, hyperspectral radiative transfer calculations via low-streams interpolation, J. Geophys. Res., 115, D10206, doi:10.1029/2009JD012803.

Osterman, G. B., et al. (2016), Orbiting Carbon Observatory-2 (OCO-2) data product user's guide, operational L1 and L2 data versions 7 and 7R, Pasadena, Calif.

Platnick, S., S. A. Ackerman, M. D. King, K. Meyer, W. P. Menzel, R. E. Holz, B. A. Baum, and P. Yang (2015), MODIS atmosphere L2 cloud product (06_L2), NASA MODIS Adaptive Processing System, Goddard Space Flight Center, doi:10.5067/MODIS/MYD06_L2.006.

Preusker, R., and R. Lindstrot (2009), Remote sensing of cloud-top pressure using moderately resolved measurements within the oxygen A band-A sensitivity study, J. Appl. Meteorol. Climatol., 48(8), 1562-1574, doi:10.1175/2009JAMC2074.1.

Preusker, R., J. Fischer, P. Albert, R. Bennartz, and L. Schüller (2007), Cloud-top pressure retrieval using the oxygen A-band in the IRS-3 MOS instrument, Int. J. Remote Sens., 28(9), 1957-1967, doi:10.1080/01431160600641632.

Rozanov, V. V. (2004), Semianalytical cloud retrieval algorithm as applied to the cloud top altitude and the cloud geometrical thickness determination from top-of-atmosphere reflectance measurements in the oxygen A band, J. Geophys. Res., 109, D05202, doi:10.1029/ 2003JD004104.

Sanghavi, S., M. Lebsock, and G. Stephens (2015), Sensitivity analysis of polarimetric O2 A-band spectra for potential cloud retrievals using OCO-2/GOSAT measurements, Atmos. Meas. Tech., 8(9), 3601-3616, doi:10.5194/amt-8-3601-2015. 
Savtchenko, A., R. Kummerer, P. Smith, A. Gopalan, S. Kempler, and G. Leptoukh (2008), A-Train data depot: Bringing atmospheric measurements together, IEEE Trans. Geosci. Remote Sens., 46(10), 2788-2795, doi:10.1109/TGRS.2008.917600.

Sherwood, S. C., S. Bony, and J.-L. Dufresne (2014), Spread in model climate sensitivity traced to atmospheric convective mixing, Nature, 505(7481), 37-42, doi:10.1038/nature12829.

Slingo, A. (1990), Sensitivity of the Earth's radiation budget to changes in low clouds, Nature, 343(6253), 49-51, doi:10.1038/343049a0

Sneep, M., J. F. de Haan, P. Stammes, P. Wang, C. Vanbauce, J. Joiner, A. P. Vasilkov, and P. F. Levelt (2008), Three-way comparison between OMI and PARASOL cloud pressure products, J. Geophys. Res., 113, D15S23, doi:10.1029/2007JD008694.

Spurr, R. J. D. (2002), Simultaneous derivation of intensities and weighting functions in a general pseudo-spherical discrete ordinate radiative transfer treatment, J. Quant. Spectrosc. Radiat. Transfer, 75(2), 129-175, doi:10.1016/S0022-4073(01)00245-X.

Spurr, R. J. D. (2006), VLIDORT: A linearized pseudo-spherical vector discrete ordinate radiative transfer code for forward model and retrieval studies in multilayer multiple scattering media, J. Quant. Spectrosc. Radiat. Transfer, 102(2), 316-342, doi:10.1016/j.jqsrt.2006.05.005.

Spurr, R. J. D., T. P. Kurosu, and K. V. Chance (2001), A linearized discrete ordinate radiative transfer model for atmospheric remote-sensing retrieval, J. Quant. Spectrosc. Radiat. Transfer, 68(6), 689-735, doi:10.1016/S0022-4073(00)00055-8.

Stephens, G., and A. Heidinger (2000), Molecular line absorption in a scattering atmosphere. Part I: Theory, J. Atmos. Sci., 57(10), 1599-1614, doi:10.1175/1520-0469(2000)057<1599:MLAIAS>2.0.CO;2.

Stephens, G. L. (1978), Radiation profiles in extended water clouds. II: Parameterization schemes, J. Atmos. Sci., 35(11), 2123-2132, doi:10.1175/1520-0469(1978)035<2123:RPIEWC > 2.0.CO;2.

Szczodrak, M., P. H. Austin, and P. B. Krummel (2001), Variability of optical depth and effective radius in marine stratocumulus clouds, J. Atmos. Sci., 58(19), 2912-2926, doi:10.1175/1520-0469(2001)058<2912:VOODAE >2.0.CO;2.

Taylor, T. E., et al. (2016), Orbiting Carbon Observatory-2 (OCO-2) cloud screening algorithms: Validation against collocated MODIS and CALIOP data, Atmos. Meas. Tech., 9(3), 973-989, doi:10.5194/amt-9-973-2016.

Vanbauce, C., J. C. Buriez, F. Parol, B. Bonnel, G. Sèze, and P. Couvert (1998), Apparent pressure derived from ADEOS-POLDER observations in the oxygen A-band over ocean, Geophys. Res. Lett., 25(16), 3159-3162, doi:10.1029/98GL02324.

Várnai, T., and A. Marshak (2002), Observations of three-dimensional radiative effects that influence MODIS cloud optical thickness retrievals, J. Atmos. Sci., 59(9), 1607-1618, doi:10.1175/1520-0469(2002)059<1607:OOTDRE $>2.0 . C O ; 2$.

Vaughan, M. A., K. A. Powell, D. M. Winker, C. A. Hostetler, R. E. Kuehn, W. H. Hunt, B. J. Getzewich, S. A. Young, Z. Liu, and M. J. McGill (2009), Fully automated detection of cloud and aerosol layers in the CALIPSO lidar measurements, J. Atmos. Oceanic Technol., 26(10), 2034-2050, doi:10.1175/2009JTECHA1228.1.

Webb, M. J., et al. (2006), On the contribution of local feedback mechanisms to the range of climate sensitivity in two GCM ensembles, Clim. Dyn., 27(1), 17-38, doi:10.1007/s00382-006-0111-2.

Winker, D. M., et al. (2010), The CALIPSO mission: A global 3D view of aerosols and clouds, Bull. Am. Meteorol. Soc., 91(9), 1211-1229, doi:10.1175/2010BAMS3009.1.

Wood, R., and D. L. Hartmann (2006), Spatial variability of liquid water path in marine low cloud: The importance of mesoscale cellular convection, J. Clim., 19(9), 1748-1764, doi:10.1175/JCLI3702.1.

Yoshida, Y., Y. Ota, N. Eguchi, N. Kikuchi, K. Nobuta, H. Tran, I. Morino, and T. Yokota (2011), Retrieval algorithm for CO2 and CH4 column abundances from short-wavelength infrared spectral observations by the greenhouse gases observing satellite, Atmos. Meas. Tech., 4(4), 717-734, doi:10.5194/amt-4-717-2011.

Zeng, S., C. Cornet, F. Parol, J. Riedi, and F. Thieuleux (2012), A better understanding of cloud optical thickness derived from the passive sensors MODIS/AQUA and POLDER/PARASOL in the A-train constellation, Atmos. Chem. Phys., 12(23), 11,245-11,259, doi:10.5194/ acp-12-11245-2012. 